\title{
Concurrent Engineering Through Product Data Standards
}

\section{Research Information Cente: \\ National Institute of Standards \\ and Techrology \\ Gathersburg, Maryland 20839}

ocy 1099

\section{Gary P. Carver \\ Howard M. Bloom}

U.S. DEPARTMENT OF COMMERCE National Institute of Standards and Technology

Manufacturing Engineering Laboratory Factory Automation Systems Division Gaithersburg, MD 20899
U.S. DEPARTMENT OF COMMERCE

Robert A. Mosbacher, Secretary NATIONAL INSTIUIE OF STANDARDS AND TECHNOLOGY

John W. Lyons, Director

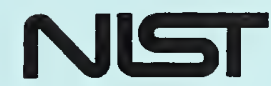





\section{Concurrent Engineering Through Product Data Standards}

\section{Gary P. Carver \\ Howard M. Bloom}

U.S. DEPARTMENT OF COMMERCE National Institute of Standards and Technology

Manufacturing Engineering Laboratory Factory Automation Systems Division Gaithersburg, MD 20899

May 1991

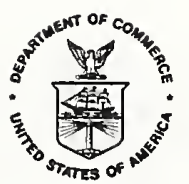

U.S. DEPARTMENT OF COMMERCE

Robert A. Mosbacher, Secretary NATIONAL INSTIIUTE OF STANDARDS AND TECHNOLOGY

John W. Lyons, Director 



\title{
CONCURRENT ENGINEERING THROUGH PRODUCT DATA STANDARDS
}

\author{
Gary P. Carver
}

\section{Howard M. Bloom}

\author{
Factory Automation Systems Division \\ Manufacturing Engineering Laboratory \\ National Institute of Standards and Technology \\ Gaithersburg, MD 20899
}

\begin{abstract}
Concurrent engineering involves the integration of people, systems and information into a responsive, efficient system. Integration of computerized systems allows additional benefits: automatic knowledge capture during development and lifetime management of a product, and automatic exchange of that knowledge among different computer systems. Critical enablers are product data standards and enterprise integration frameworks. A pioneering assault on the complex technical challenges is associated with the emerging international Standard for the Exchange of Product Model Data (STEP). Surpassing in scope previous standards efforts, the goal is a complete, unambiguous, computer-readable definition of the physical and functional characteristics of a product throughout its life cycle. U.S. government agencies, industrial firms, and standards organizations are cooperating in a program, Product Data Exchange using STEP (PDES), to develop and implement STEP in a shared-database environment. PDES will lead to higher, integrated levels of automation based upon information standards and frameworks. U.S. manufacturers will benefit from concurrent engineering without sacrificing the historical strengths and traditions of individuality, initiative, and intellectual property rights. Concurrent engineering, through information technology and standards, represents the power of a new industrial revolution. The role of the NIST National PDES Testbed, technical leadership and a testing-based foundation for the development of STEP, is described.
\end{abstract}

\section{KEY WORDS}

Automated manufacturing; concurrent design; concurrent engineering; information standards; manufacturing standards; PDES; product data engineering; product data sharing; product data standards; STEP 
This manuscript will appear as a chapter in Control and Dynamic Systems: Advances in Theory and Applications, Vol. 35, "Advances in Manufacturing and Automation," C.T. Leondes, ed., Academic Press, San Diego, CA. 


\title{
CONCURRENT ENGINEERING THROUGH PRODUCT DATA STANDARDS
}

\author{
Gary P. Carver
}

\section{Howard M. Bloom}

\author{
Factory Automation Systems Division \\ Manufacturing Engineering Laboratory \\ National Institute of Standards and Technology \\ Gaithersburg, MD 20899
}

\section{INTRODUCTION}

Product data standards will revolutionize U.S. manufacturing and enable U.S. industry to build on its traditional strengths and regain its competitive edge for the twenty-first century. Standards will enable concurrent engineering to be utilized in the diverse, dynamic and heterogeneous multienterprise environment that traditionally has characterized U.S. industry.

Concurrent engineering provides the power to innovate, design and produce when all possible impacts and outcomes can be considered almost immediately. It is the use, in all phases of a manufacturing activity, of all the available information about that activity. It represents the commonality of knowledge applied to a production goal.

Concurrent engineering can stimulate and maintain the diverse and individualistic nature of the entrepreneurial environment by expanding access to knowledge. It forces a global optimization among all of the product life cycle processes within a design and production system.

However, in an automated environment, concurrent engineering is impossible without standards. That is, the full automation and integration of industrial processes is impossible unless standardized hardware and software, especially standardized knowledge and knowledge models, exist to allow intercommunication among all types of computerized systems. The significance and potential impact of this assertion are the subjects of this document. 
In principle, concurrent engineering does not have to be an automated process; it could be people interacting directly with other people. In practice, in today's manufacturing environment, the increased complexity of products and processes and the use of computerized systems precludes sole reliance on people-to-people concurrent engineering. The approach to concurrent engineering has to be through the automatic sharing of knowledge by computerized systems. It can be thought of as automated concurrent engineering, or computer-aided concurrent engineering.

In the U.S., the introduction of concurrent engineering to an enterprise, or to a group of connected enterprises, through people-to-people interactions requires usually unacceptable cultural changes. Because it emphasizes teamwork rather than competition, people-to-people concurrent engineering may be in conflict with a company's culture or management style. Or it may interfere with established relationships among the departments within a company or among the companies within a group of companies.

However, introducing concurrent engineering through integrated computer systems does not require cultural changes. Even while the integrated computer systems are sharing information, people in the manufacturing environment have the choice of how they respond to the information presented to them automatically by their computers. They do have to alter the way they work because they are utilizing greater amounts of information; however, they do not have to alter the way they interact personally with other people. In this manner, concurrent engineering does not require cultural changes. People and companies can interact and can perform their activities either individually or collectively, whatever style suits them. The entrepreneurial spirit does not have to be stifled by business-imposed interactions. The key is that the computer systems used by the people and companies interact effectively, and automatically.

Concurrent engineering achieved through the integration of computer systems can create a cooperative environment within a company, as well as among companies. In fact, "multi-enterprise concurrent engineering" can result in bringing together independently innovative companies without any loss of independence. This will provide the mechanism for the U.S. to develop its own, unique, U.S. culture-based approach for achieving world-class manufacturing.

If the approach to concurrent engineering is through automation, concurrent engineering requires the application of information technology to create the means for automated systems to communicate and interoperate. For example, within a manufacturing enterprise, computer-aided design systems must be able to share information with analysis systems, manufacturing systems, and distribution systems. Eventually, concurrent engineering can be applied to all business systems, not only manufacturing systems.

Interconnected automated business systems will provide managers, engineers, accountants, marketing specialists, distributors, and everyone involved in a business enterprise with all the information they need to carry out their functions. This includes information they need to make decisions as well as information about how their decisions affect the decisions and activities of everyone else in the business. Plans and actions will be made simultaneously, without the delays experienced in traditional paper communications and face-to-face meetings as projects progress step-by-step in linear fashion.

Even suppliers, partners, and customers can be linked through an information network. In this way, multi-enterprise concurrent engineering can create vertically or horizontally integrated manufacturing 
entities de facto. Although the suppliers would not be controlled by the systems integrator, for example, as they might be in a vertically integrated entity, supplier companies and systems assembly companies might cooperate to their mutual advantage through the sharing of product data and decision-related information.

In our increasingly global economy, digital information technology has emerged as a critical determinant of international competitiveness. From computers to telecommunications to military systems to consumer electronics, the future of a nation's economic and worldwide influence will depend on its excellence in digital information technology. Just as the industrial revolution changed the world order, the information revolution will too. Just as steel, ships, and computers affected the balance of economic and military power, information technology will too. Concurrent engineering is one of the applications of information technology that will provide unique economic opportunities.

The result of multi-enterprise concurrent engineering is more than just the optimization of a design and production system--it is a broader optimization of an industrial system. The technical challenges are numerous and difficult. Equally challenging is the attainment of international consensus on the methods for achieving the required networking of diverse types of business systems. International consensus on the means for integrating automated systems--the standards--is essential. No single company, in fact no single country, has enough resources to develop suitable methods applicable to all businesses in all countries. Even if it were to happen that one company developed an integration method, the likelihood of acceptance by everyone else is negligible. Clearly, the best approach is through consensus-based international standards.

Yet sometimes standards are viewed as constrainers of innovation and inhibitors of new technologies. Fortunately, standards for enterprise integration are interface standards or "open system standards." Interface standards relate to interoperability, including data exchange and intercommunication, among different hardware and software elements. Interface standards encourage independent development of interoperable products because they specify both the characteristics of critical interfaces and the way in which the information transferred across the interfaces is represented digitally.

Such standards are welcomed by manufacturers because they lower barriers to market entry and they enlarge the market. From the customer's view, open system standards lead to more intense competition, a larger number of vendors from which to choose, a greater variety of off-the-shelf solutions that are both less likely to become obsolete and more likely to be easily integrated into existing systems, modular systems that can be configured for improved performance in a specific application, and, as a result, lower prices. Manufacturers do not want to venture down proprietary paths with the risk that they may one day find themselves at a dead end. Everybody wins.

Concurrent engineering, based upon product data standards and enterprise integration framework standards, truly represents a new form of concurrent engineering that can be called "multi-enterprise concurrent engineering." Multi-enterprise concurrent engineering extends the principles of concurrent engineering to our U.S. environment. It can be defined as the systematic approach, across industrial enterprises, to the integrated concurrent design of products and their related processes (such as manufacturing and support) through the sharing of product data.

Achieving the benefits of concurrent engineering (Section II) requires an understanding of the unique role of the design process in the life cycle of a product (Section III). However, concurrent 
engineering encompasses more than the individual processes in the life cycle of a product. It also includes social practices and customs among people and their organizations that are involved in those life cycle processes. Fortunately, it can be shown that concurrent engineering practices implemented using integrated automated systems will not interfere with traditional social interactions but will greatly enhance the strengths of the U.S. style of commerce (Section IV). The essential ingredients for this to happen are the technologies and standards that will allow the sharing of information among all computerized business systems. An unprecedented effort by a variety of organizations to develop the required technologies and to implement internationally the required product data standards is underway. STEP, the Standard for the Exchange of Product Model Data, is the focus of that effort (Section V).

\section{CONCURRENT ENGINEERING IS TEAMWORK-IN- EFFECT}

Concurrent engineering is a process that involves the integration of information. In principle, in a concurrent engineering approach, all the available information about a product is accessible at every stage in its design, manufacture, support, and recovery or disposal, as illustrated in Figure 1.

A concurrent engineering approach could be implemented by assembling a team of (human) experts, each of whom is a specialist responsible for one or more stages of the product's life cycle. The team would create and support the product over its life. Access to information would be accomplished either by request for the information by the expert who recognizes the need for it or by contribution of the information by the expert who recognizes its usefulness at the time. The human team is the mechanism for integrating the product information. Examples of product information used by experts in different phases of a product's life cycle are shown in Figure 2.

The human team approach works, but is limited by cultural and organizational practices and by the amount and complexity of the information. An alternative approach that has none of these limitations is automation of the creation-to-disposal process for a product. In this approach, computerized systems access the information and either automatically utilize it or offer it to the appropriate human specialist at the proper time. Therefore, the mechanism for integrating the product information is the totality of interconnected, information-sharing automated systems. This is illustrated in Figure 3. No human team need meet or interact face-to-face. The specialists could be separated both physically and organizationally.

Of course, human specialists must still play a role. They create designs, make value judgements, and make decisions from information provided by the automated systems.

Nevertheless, concurrent engineering truly represents teamwork, even in its automated embodiment, when an actual team of human experts has not been created. Using a concurrent engineering approach made possible by integrated automated systems, product experts operate as they would in a traditional environment. This is because they (or their computers) utilize information from and provide information to each other as needed. 


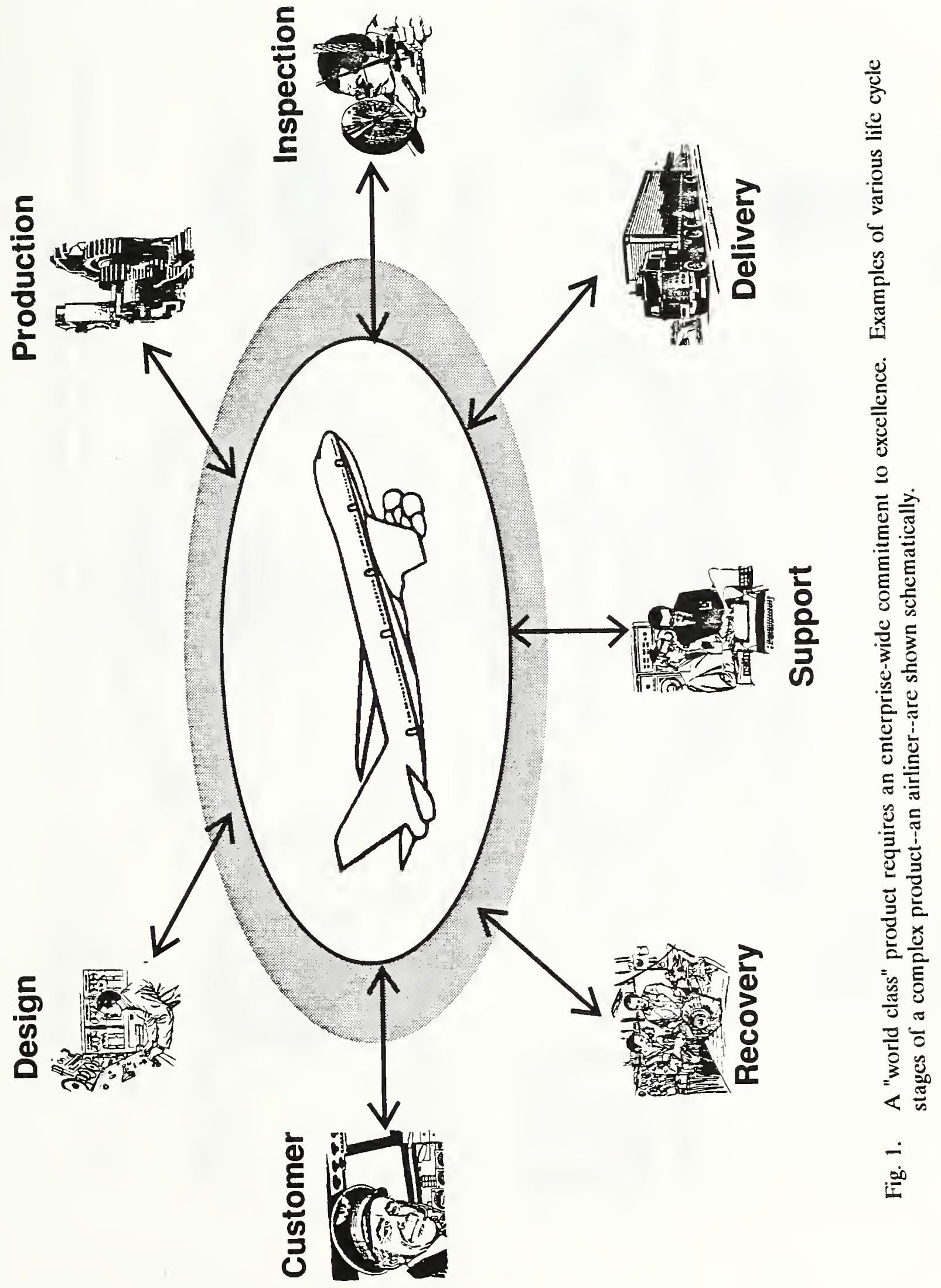




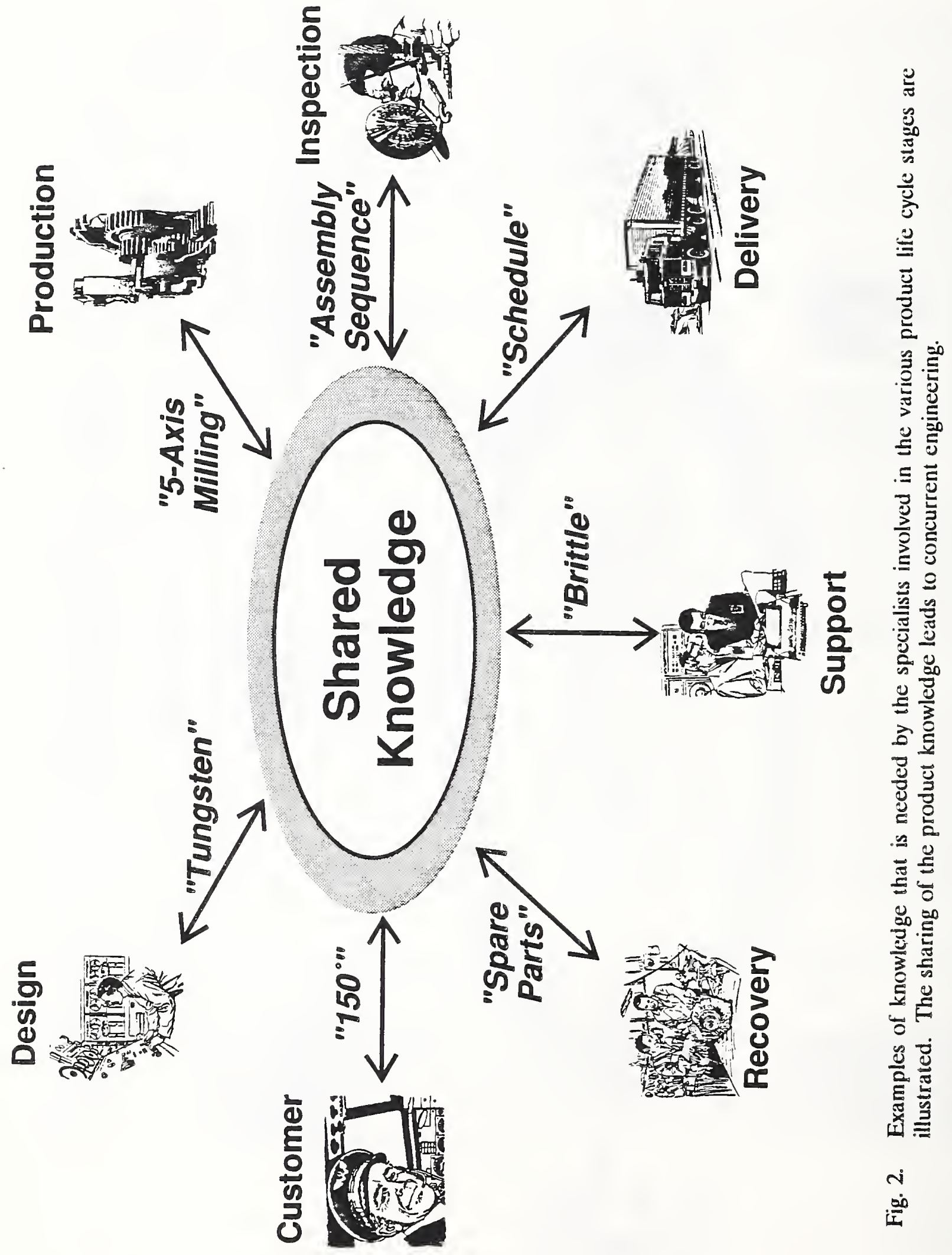




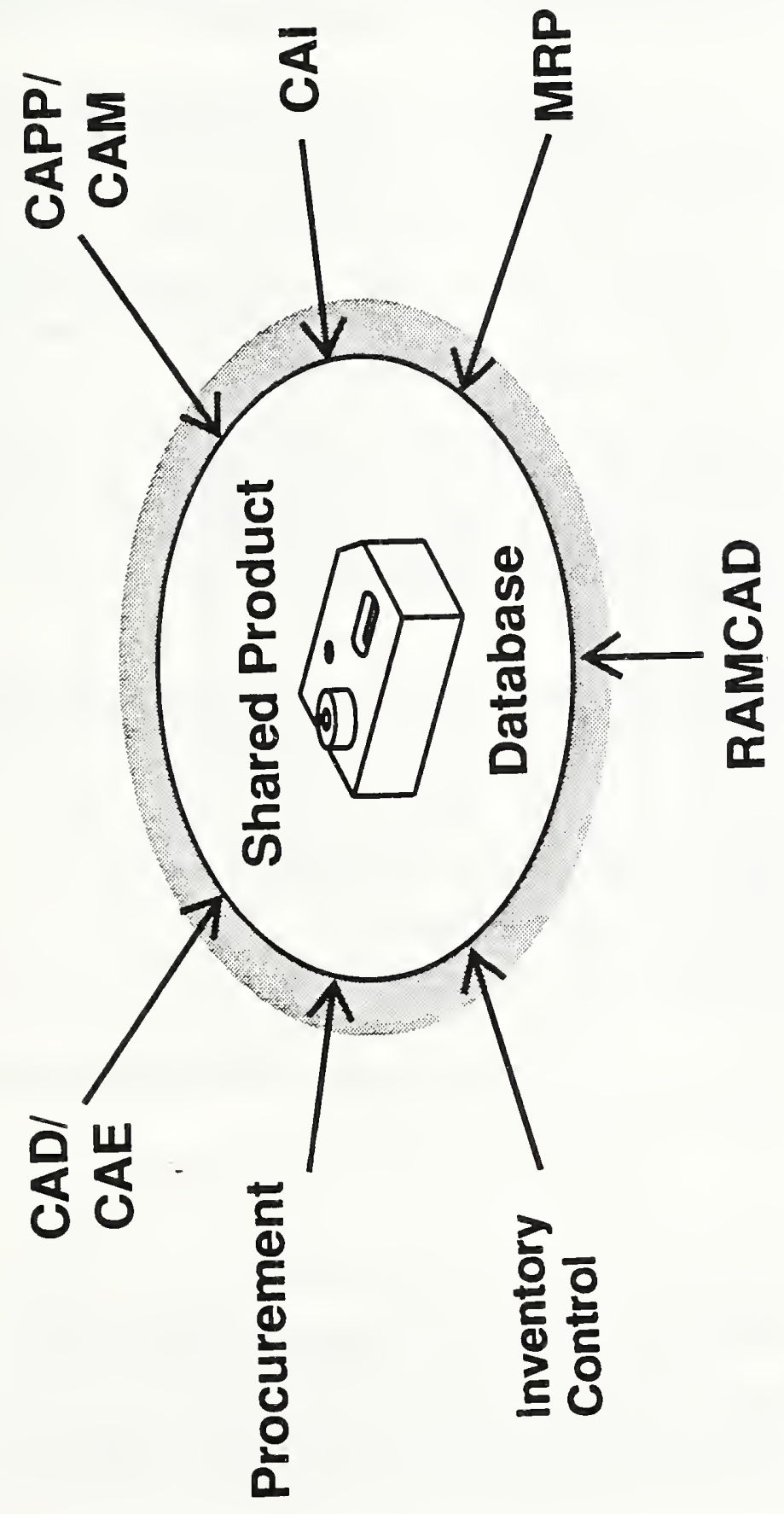

를

을 의 을 뜽를 잉 을

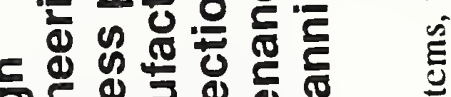

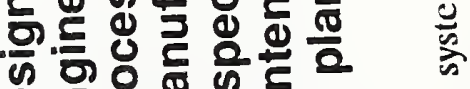

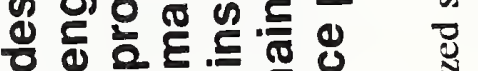
웅ㅇㅇㅇํㄹ ఏ웡 Ð ส

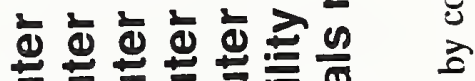

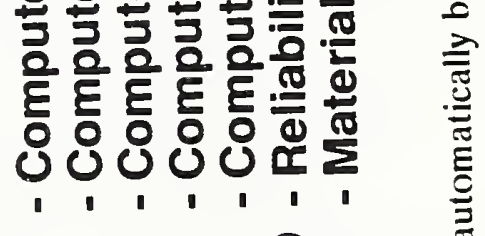

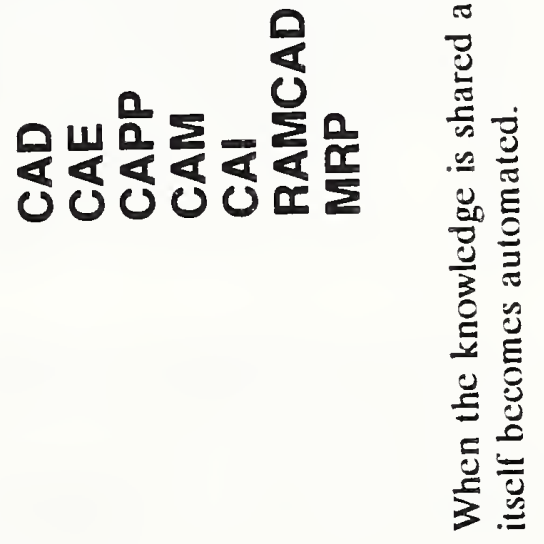
$\dot{m}$ 这 
Concurrent engineering is an old concept. It has sometimes been called concurrent design, simultaneous engineering, and system engineering. Even prior to these labels, in earlier times when individual craftspeople created individual objects, they took into account such factors as the properties of the materials, the manufacturability of the parts, and the function and utility of the object. The integration of the product information occurred within the mind of each individual craftsperson. The end result was a complete product, ready for use by the customer.

When factors such as technology led to more complex products as well as specialization and compartmentalization among experts and workers, the integration of all relevant information was no longer spontaneous. Increasingly, the tendency was that the information was made available sequentially: the designer designed, then the manufacturer manufactured, and so forth.

In contrast, concurrent engineering is an inherently parallel process. The integration of the information required for all phases of the life of a product represses serialism and promotes parallelism. A formal definition of concurrent engineering emphasizes this idea [1]:

"Concurrent engineering is a systematic approach to the integrated, concurrent design of products and their related processes, including manufacture and support. This approach is intended to cause the developers, from the outset, to consider all elements of the product life cycle from conception through disposal, including quality, cost, schedule, and user requirements."

It is apparent that in today's U.S. manufacturing environment barriers must be overcome to realize concurrent engineering. Therefore, in practice concurrent engineering may mean:

- Overcoming resistance to teamwork; that is, getting designers,

manufacturing engineers, and support personnel to work together,

- Overcoming competitiveness between individuals,

- Retraining the educationally specialized in newer technologies,

- Modifying management styles and organizational cultures, and

- Developing new types of computer-based tools.

These are input- or investment-oriented issues. Looking instead at what concurrent engineering means in terms of outputs or benefits, concurrent engineering may mean:

- Lower costs,

- Shorter time-to-market, and

- Greater quality.

These results will affect the survival of a company or the success of an industry in world markets.

While any and all of these issues are real (and are explored later in Section IV), the single most important issue regarding concurrent engineering is that standards for all types of enterprise 
information--especially product data standards--are essential because concurrent engineering is impossible without standards.

Even if a multidisciplinary team of engineers were assembled to produce a product as well as the procedures necessary to maintain it in use, it is impossible such a team could operate effectively without the ability of the automated systems to communicate and share information about all phases of the product's existence. In today's manufacturing environment, concurrent engineering means integrated information systems--and that means product data standards are needed.

\section{B. The Need for Concurrent Engineering}

Competitive success depends on shortening the time between conception and introduction of new technologies and products into the marketplace. To meet the need to minimize time-to-market, computer-aided tools are used to move the product from concept through design, prototype, manufacture, test, and introduction into the marketplace (from concept to consumption).

Even as pressure is applied to decrease product development time, the diversity of activities and expertise required to bring a product to fruition are increasing dramatically. This is because in addition to meeting functional needs, the product must meet energy, environmental, health and safety, and other requirements. These non-traditional requirements are becoming more demanding on manufacturers as the sophistication of our culture advances and as our knowledge of the impacts of human activities on the global environment expands.

Because of the need to minimize the time from conception of a product to its delivery to a customer, and because of the amount and variety of information needed by manufacturers, computers are essential in manufacturing. They are used to design products, plan for their manufacture, control the equipment that produces them, control the equipment that tests them, manage their distribution, and help support their operation, repair and maintenance. Furthermore, because most manufacturers of complex products, for example, vehicles and computers themselves, manufacture only a fraction of the parts in these products, there are needs relating to activities such as inventory control, scheduling, and ordering, as well as the coordination of all the manufacturing activities of the supplier companies. But the needs can be only partially met, and the advantages of using computers only partially achieved, unless the computers can interoperate--that is, share information-so that they can perform their tasks in parallel. In this manner computers and integration naturally point to--even demand--concurrent engineering.

\section{The Benefits of Concurrent Engineering}

Concurrent engineering can shorten the time required before a product is marketed. It can improve productivity, profitability and competitiveness. It can lower costs, reduce waste, improve quality, and improve efficiency in all phases of the life of a product [1]. It can allow suppliers and vendors to coordinate their operations. It can enable manufacturers to cooperate in consortia in precompetitive projects. Such coordinated operations increase the resources, lower the cost and reduce the risk for each individual participant. This may allow the participants as a group to be more innovative and risk-taking to produce more competitive products. In today's aggressively competitive world market, concurrent engineering may mean the difference between failure and success of an industry. 
The economic benefit of success (that is, at least survival) compared to failure is obvious. But there are other benefits related to overcoming the barriers:

Teambuilding. Concurrent engineering integrates, through the sharing of information, all the people involved in a manufacturing activity. They become a team through their automated systems. The interactions can be optimized, even though they may not include face-to-face interactions, and the individual team members can perform to the limits of their capabilities without changing their personal or interpersonal styles.

Workers' Career Growth. New forms of workplace organization in modern manufacturing facilities gives workers more responsibility [2]. Increasingly, they must use judgement and make decisions. Concurrent engineering will accelerate this trend, and it will support workers with the information they need.

Management. The concurrent engineering environment will give managers the ability to oversee all activities throughout their enterprise--without any additional bureaucracy. Managers will be able to have the information they need, as it is created, to anticipate, plan, and act quickly.

Competition and Choice. It takes years for companies in an industry to recognize all of the different specialty niches for systems and to develop viable products. For example, although the basic interface specifications for personal computers were established in the early 1980's, new types of hardware and software products are still being defined today. In the same way, the integrated concurrent engineering environment will provide opportunities for new products that cannot be predicted now. The enabling vehicles are standards. In addition, since no one manufacturer offers computers that will design, manufacture, and support a product, interface standards are essential.

Additional thoughts on the benefits of concurrent engineering as both a method and an environment are discussed in Section IV. In the next section, an important reason why concurrent engineering can provide considerable benefits is discussed. The reason is that concurrent engineering affects design decisions. Concurrent engineering, based upon integrated automated systems, allows specialized engineers from all phases of a product's life cycle to participate in design decisions, and this has a major impact on the cost of the product [3].

\section{DESIGN IS THE CRITICALARENA FOR CONCURRENT ENGINEERING}

If manufacturing is the use of energy to convert materials and components into saleable products, then design is the use of knowledge to convert information and requirements into functionality. Design includes both design of a product as well as design of the manufacturing systems and processes to produce the product. Design decisions affect all aspects of the life of a product, including production cost and other characteristics of the product's manufacture, marketing, maintenance, repair, and disposal. A "good" product design addresses the concerns of each of these characteristics as well as the quality, cost, and functionality of the product to the user. Examples of the kinds of information needed by a designer are listed in Figure 4 . World-class products, products that are competitive in timeliness, performance, cost, and quality, result from good design. 
The term "concurrent design" refers to the "coordinated design of products and processes so that effective and efficient manufacturing will be possible" [4]. Concurrent design is therefore consistent with intuitive concepts of "good" design. (Although good engineering design has been more formally described as a process that ensures "products are designed and manufactured with 'designed in' instead of 'tested in' reliability and maintainability" [5].) The point is that concurrent design is the only design approach that works in a concurrent engineering approach.

\section{A. The Uniqueness of Design in the Life Cycle of a Product}

Possibly because it includes the initial stages of the development of a product, and certainly because it determines the nature of the future attributes of a product, the process of design exerts the most control over a product's life cycle. For example, about $60 \%$ of a product's cost is fixed very early in the process of design; overall, the design process may fix as much as almost $90 \%$ of the total cost of a product [6]. This means that production and production management decisions affect only about $20 \%$ to $30 \%$ of the total costs. This is shown in Figure 5. Despite its importance, the design process is often inefficient, detached from the production process, undocumented in terms of the rationale for design decisions, and production-facility dependent.

A fundamental problem with developing effective design environments and representing design intent has been a lack of a conceptual model of the design process. Without a model for the various facets of the design process, computer-based design tools will remain customized and relatively isolated, interacting only at low levels. In this situation, unique solutions and individualized integration schemes substitute for an appropriate architecture for the use of concurrent engineering in design.

The design process can be divided into conceptual design, detailed design, and manufacturing system/process design. Early in the conceptual design phase, just when there is the most flexibility, most of the total costs of the product are committed.

\section{Conceptual Design}

The conceptual design phase is when the concept for the product, including sub-assemblies and components, is developed. The general shape of the product is known, but detailed geometric information is not yet available. The output from conceptual design activities contains assumptions, constraints, conditions, and other information related to a product that must be used by downstream operations to produce and support the product.

Important decisions are made during conceptual design that affect the nature of a product, such as complexity and maintainability. The computer-aided engineering (CAE) tools required to involve design in the overall concurrent engineering approach should be integrated and interfaced and should be capable of representing functional information about the product. The designer needs such information early in the design process, and in a form that is readily accessible to the design tools being used.

Computer-aided engineering tools allow the designer to model the qualitative and functional performance of the product. CAE tools include tools for simulation of operations, structural and mechanical analysis such as finite element and boundary element analysis, fluid flow and thermal 


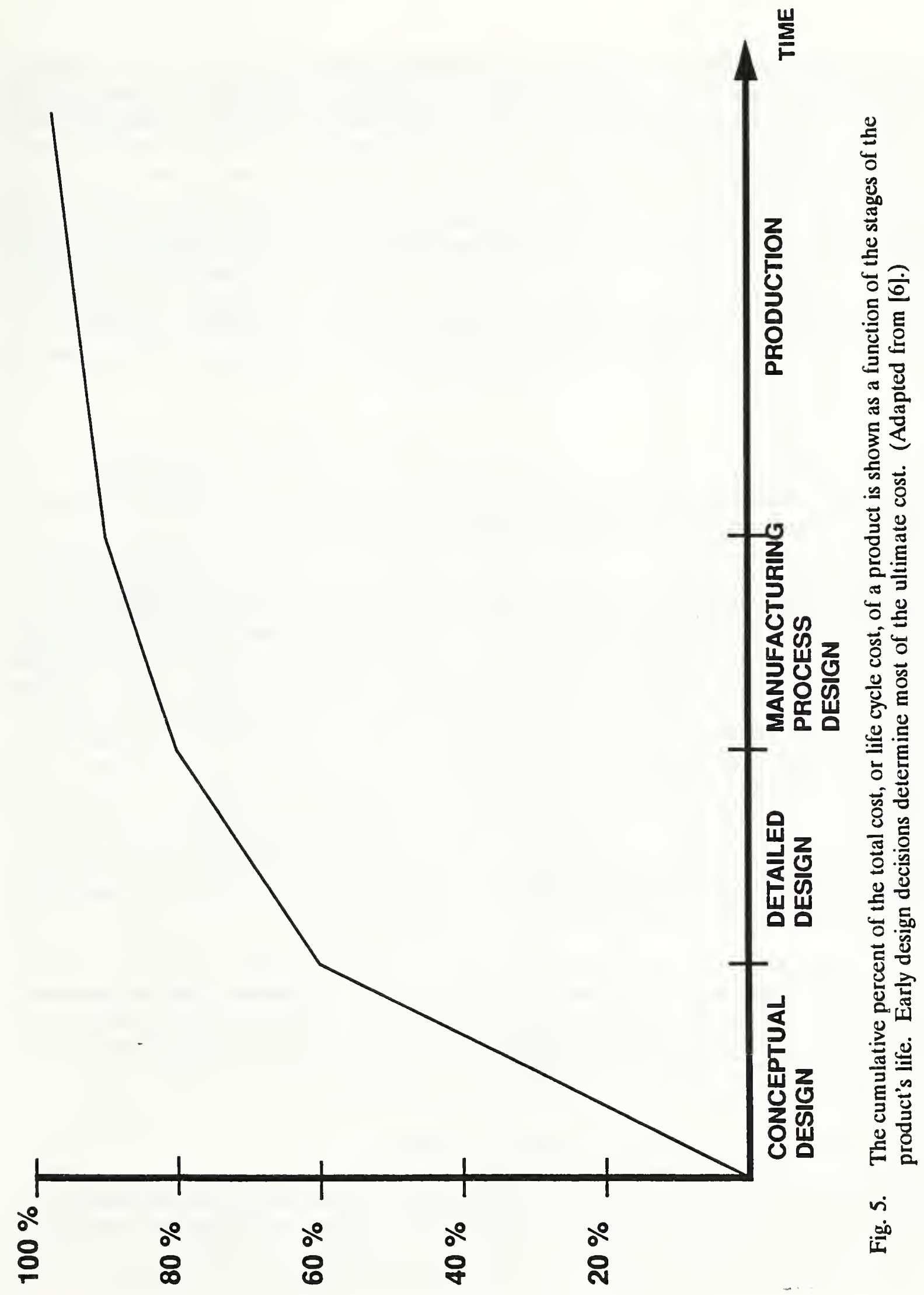

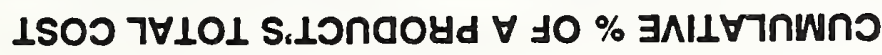


analysis, solid and surface modeling and a variety of other specialized evaluations of the potential product. Such analyses to determine performance characteristics are executed usually on an idealized geometry. Unfortunately, most of today's CAE tools have their own proprietary data structures and interfaces. The data are not available to other computer programs and are interfaced only to the human user.

In addition to lacking the ability to exchange information, most of today's CAE tools also lack the ability to represent non-geometric functional information about the product. It is important to know, for example, whether a product must be non-conductive or corrosion resistant and whether it will be used in conjunction with or interact with some other device. To allow for automated or computer-sensible concurrent engineering, CAE tools must be able to capture function information and to represent physical objects better than is possible today.

\section{Detailed Design}

During the detailed design phase the product is specified completely and unambiguously. The geometry and topology, the dimensions and tolerances, and any features needed as a result of the analyses performed during the conceptual design phase are specified in detail.

In addition to specifying the product in detail, assurance must be developed that all features of the product design are consistent with priorities and considerations for downstream life cycle stages. For example, it must be determined that tolerances are neither too loose to meet functionality needs nor too restrictive for manufacturability and low cost. Tolerance decisions reflect the capability of available manufacturing equipment; either the designer modifies the design to accommodate equipment-dictated tolerances or designs-in tighter tolerances that necessitate specialized equipment. To create a design having predictable product functionality, manufacturability and cost, the designer must know the effects of alternative design decisions.

The designer also needs information about the importance of the product attributes as they relate to the processes the product will encounter in its life to make knowledgeable design decisions. Questions such as, "Is performance more important than maintainability?," "Is ease of assembly more important than durability?," and "How do these characteristics relate to cost?" must be considered and must have answers if detailed design is to be completed successfully.

In a concurrent engineering environment, the design tools have access to information about downstream life cycle considerations, including costs. Furthermore, the tools are able to measure and express in a quantifiable manner the results of analyses of the impact of detailed design decisions on downstream stages of the life cycle. Obviously, the CAE tools must be interfaced to other automated systems if they are to accomplish such tasks.

\section{Manufacturing System and Manufacturing Process Design}

The design of the manufacturing systems and processes that will be used to produce the product involves detailed knowledge of a different type than may have been needed earlier in the design process. Information such as the number of devices to be produced, the batch size, the process capabilities, the availability of materials, and other information about manufacturing operations and 
equipment was needed earlier, but typically only to avoid a design that violated certain practical limitations on its production. At this point, this kind of information is needed to determine the actual sequence of manufacture and the facilities and production equipment required.

The CAE tools used at this point are also different than those used earlier in the design process, but they share the same need for integration and interfacing. Software for process planning, modeling of the actual manufacturing processes, programming and controlling the production machines, fixturing the machines, and other production operations must have access to product design information.

It is easy to see how tolerance information would be important because it relates to the production processes and their capabilities for precision. What is less easy to understand is how design intent information is critical for concurrent engineering. This is considered in the next section.

\section{B. The Representation of Design Intent}

Solid modeling systems improved the design process when they replaced "wireframe" drawings. Solid modeling systems can be used to represent an object being designed accurately and unambiguously. Recently, solid modeling systems have been used to generate finite element meshes and to generate tool paths for machining. However, solid models still do not help in all stages of the product life cycle. They only help to provide more complete geometric information. The goal for concurrent engineering is product models that include not only dimensions and tolerances, as well as other feature information, but also information about the decision-making process that led to a product design.

To maximize the benefits of concurrent engineering, the various product life cycle systems must be able to determine how, for example, the shape or dimensions were derived and why the part must be non-conductive. Unless such design-intent information is available, it is difficult to minimize the risk of making modifications and improvements to products and manufacturing and repair processes.

New software tools and models are needed to measure such properties as design "goodness;" manufacturability; product and process performance; product and process costs; impacts of changes from one alternative design to another; and manufacturing configuration. The following are some examples of needed design tools and models [1], [7]:

Process models for various manufacturing processes such as metal cutting, forming, injection molding, and casting. The models can be used to alter product geometry or process parameters through various engineering, geometric, statistical, and scientific analyses. The models can provide a measurement (such as tolerances, material integrity, strength, and surface finish) for projecting process performance (such as speeds, temperatures, and precision).

Assembly and cost models to provide the designer with cost prediction estimates and design options for more easily assembled products. Geometric models of position and path for product handling are also useful. 
Manufacturing system models to measure the capacity of systems. The integration of capacity and capability models in manufacturing allows the designer to simulate and analyze the manufacturing processes, the process technologies, and the design of statistical quality control methods.

Factory engineering models to provide the data required to configure new factories. These tools can be used to show how a decision to improve one process in the factory might result in simultaneous changes in the data maintained in several associated applications. Some changes which might occur automatically include revisions to plant layout drawings, utility requirements, simulation models, cost/payback projections, and procurement specification documents for the proposed system.

Future systems will be "intelligent machines" that receive product descriptions and automatically interpret them and perform the appropriate machine operations to achieve the desired product geometry, tolerances, and material specifications. The keys are the integration of the design database into the manufacturing facility and the integration of methods for translating design descriptions into manufacturing process and control programs.

The process of design, as well as manufacturing generally, will be improved by the development of standard ways for representing design intent. Software tools will need to include a better understanding of the design process. The goal must be the inclusion of product data models that incorporate design intent information. Only with a standard representation for such information can the output of design systems be used by the variety of other automated systems responsible for a product during its life.

It should be clear that design is a process that involves a series of activities. During the design process, a large problem is decomposed into a series of smaller problems, partial solutions are proposed, and, through feedback and the resolution of constraints or tradeoffs, partial solutions are refined until an overall solution is reached.

Present computerized aids merely help speed up the design process by performing quickly the computations needed for the partial solutions. To achieve a concurrent engineering environment, a framework based upon a model of the design process and appropriate interface and product data standards is required for the integration of computer-based tools. One approach is to view the concurrent engineering environment as a "large-state machine" within which changes in the state of the system can occur in predetermined ways [8].

\section{CULTURE AND CONCURRENT ENGINEERING CAN BE COMPATIBLE}

Our current information-based society [9] seems to be characterized by the computerization of everything. Early in this period, some people feared that computerization was equal to dehumanization. However, it is now clear that it is the way computers and digital systems are implemented that can either limit or extend human interaction and control or that can either diminish our rights or endow us with additional freedoms. 
Computers seem to be the most visible sign that as the world is changing, cultural and traditional values are coming into conflict with technology. Yet there is evidence that computers can be used to preserve or even restore traditional approaches--at least at the human-machine interface. In a fundamental sense, the term "user friendly" implies a recognition of and deference to human ways of doing things. The concept of "artificial intelligence" connotes an imitation of the human mind's ability to process information. These are examples of computers helping to extend our behavior and abilities.

The relevant question is: "Does concurrent engineering represent an attack on human cultural values--such as creativity, individualism, and pride--or does concurrent engineering allow us to extend our abilities and do more of what we did before, in the way we do it best?"

The answer is that concurrent engineering is in harmony with us as humans and with our culture. Furthermore, concurrent engineering may be the only way to preserve our style of doing business in the U.S.

\section{A. The Relationship Between Concurrent Engineering and Competitiveness}

The U.S. loss of competitiveness, shrinking markets, and trade deficits are symptoms that something new is going on in the rest of the world. There can be little doubt that today the U.S. is losing world market share and technological leadership in most of the technologies important to maintaining our standard of living [10]. According to a recent government report on electronics, a major growth area in the U.S. economy in terms of employment, output, exports, and innovation and also vital to national defense and security, U.S. leadership is under serious challenge and may soon be eclipsed [11].

Another government report begins a summary in chapter one with the declaration: "American manufacturing has never been in more trouble than it is now" [12]. According to this report, manufacturing is weak because its technology is weak. And unless this weakness is cured, the U.S. will not be able to enjoy rising living standards and the continued creation of jobs at the same rate as in the past. This includes not only jobs created in the manufacturing sector, but also indirectly in the service sector. Manufacturing technology is identified as the key to national competitive success.

There can be little doubt that in today's economic climate, and for the foreseeable future, industries must compete in world markets. Among industrialized nations, international competition is increasing. Yet, as evidenced by the trade deficit, many U.S. industries are not competing successfully.

A number of factors are cited that contribute to the current difficulties of U.S. manufacturers in global markets. The factors often include federal budget deficits, low personal savings rates, the high cost of capital, the low "patience of capital," the weak dollar, short-term profit goals of corporate managers, short-term profit goals of investors, lack of a trained workforce, lack of access to foreign markets, "dumping" by foreign companies, product liability and litigation, inadequate foreign protection of intellectual property rights, federal regulatory restrictions, poor management-labor relations, foreign manufacturer-supplier relationships, antitrust laws,... Seldom, if ever, do the reasons given for U.S. poor competitiveness include either technology or standards issues. This may 
be because the role of technology and its relationship to economic factors may not be well understood. Technology is sometimes viewed as only the creation of new products rather than the improvement or revolutionary changing of existing manufacturing practices. As mentioned in the introduction, standards are sometimes viewed as inhibiting change rather than enabling or facilitating innovation and competition.

Technology can make a difference when it provides the means to build a new national economic strength or to build on an existing strength. In these ways technology can provide the means to overcome non-technical barriers and to produce a new basis for competitiveness. Completely new technologies can even change the significance of the non-technical barriers, making some of them inconsequential. Concurrent engineering based on integrated automated systems may be able to do both.

Actually, concurrent engineering has already been shown to be able to contribute to competitiveness. A Harvard Business School study showed concurrent engineering to be responsible for a $30 \%$ decrease in the time-to-market for a new car in the Japanese automotive industry [12], [13]. Even in the U.S., in a study of six defense contractors, concurrent engineering has already been shown to reduce costs $30 \%$ to $60 \%$, to reduce development time $35 \%$ to $60 \%$, to reduce defects by $30 \%$ to $80 \%$, and to reduce scrap and rework by $58 \%$ to $75 \%$ [1]. However, concurrent engineering, along with integrated computerized systems and appropriate standards, can make even more significant improvements.

\section{B. The Relationship Between Concurrent Engineering Practices and Alliances Among Businesses}

Manufacturers are linked in a chain, sometimes called a "food chain," to their materials and parts suppliers and to their customers. It would seem that close links and stable relationships would be advantageous.

In 1988, a deteriorating trade balance between the U.S. and Japan reached a deficit of \$6 billion. In 1989, for the first time, the U.S. was a net importer of computers. In 1990, also for the first time, the computer systems industry had a zero trade balance. One important reason for these trends may be cultural. The perception is that while U.S. industry is considered to have better design skills, it does not have the business partnerships and long-term strategies for commercial success. The Japanese, who lead in rapid and integrated "design for manufacturability" and in flexible manufacturing, have strong and stable business partnerships as well as long-term strategies.

It has been argued that the advantages of Japanese companies in world markets are due primarily to their diversified, vertically integrated structure and their long-term partnerships in financialindustrial groups called "keiretsu" [14]. Typically, a major bank is one member of a keiretsu. This is important because today the size of the investment necessary to develop a new technology is prohibitive to most companies. The ability of a company to develop a technology and quickly bring to market a product that utilizes that technology is an obvious advantage. This can happen when a partner company with strong consumer product development skills and effective marketing networks develops the product even as the technology is being developed. In a sense, this is multienterprise concurrent engineering. 
Yet given the evidence, U.S. industry has been slow to form cooperative relationships. Although more companies are forming consortia, especially for joint research and development in precompetitive technologies, and more companies are cultivating closer relationships with their suppliers, there is still resistance to business alliances in U.S. industry. This is true even though the interpretation of anti-trust regulations is gradually weakening and federal funds are becoming available for consortia in generic and precompetitive technologies.

The individualistic entrepreneurial spirit helped bring us to where we are today. But unless something changes, that same characteristic will prevent the U.S. from reversing its decline in competitiveness. This is because in a world "where R\&D and commercialization costs can exceed $\$ 1$ billion for a single technology, small companies working independently cannot compete. [The fragmentation of U.S. industry into small companies]...spreads R\&D funds thinly, slows technology diffusion, and diffuses manufacturing and marketing power" [15]. Yet U.S. technology companies do not make the strategic partnerships that Japanese companies do and, if the current trade, market share, and direct investment (Japanese companies buying high technology U.S. companies) statistics continue, small innovative U.S. technology companies may not be able to survive.

The behavior of U.S. companies reflects their cultural environment. "Rugged individualism" is a desirable characteristic. Strengths include creativity, innovation, and individualism, even--perhaps especially--in commerce. Competition has been a primary motivator and strength of our system; but it does not coexist well with the notion of entrepreneurs working together. The "not invented here" syndrome and the stereotypical "throw it over the wall" style among departments within companies is symptomatic of cultural values. In addition, the tendency toward an adversarial relationship can divide management and labor.

How can all this be reconciled with the need to cooperate to form large, internationally competitive business structures? How can it possibly support concurrent engineering?

The answer is that the creation of an automated interconnected computer environment can free people and companies to operate however they please. If information can be shared automatically and the computer systems people use to do their work can interact and the mechanisms are in place to create a concurrent engineering environment, then individuals can perform their activities in their own way. Instead of being constrained by having to interact and cooperate with others, people can, if they choose, be independent and work alone. Their data will be integrated. In this way, the full automation of the industrial environment will provide the needed teamwork. The entrepreneurial spirit does not have to be stifled by business-imposed interactions. Independent innovators can continue to be independent--at least in the way they operate personally.

Consequently; concurrent engineering and all it represents is not necessarily antagonistic to our culture. In fact, the creation of a concurrent engineering environment through information technology can free people and businesses to do what they do best. In the U.S., concurrent engineering can provide the cooperation that allows us to continue to be independent entrepreneurs. Concurrent engineering can meld a large number of small, highly specialized, vibrant and dynamic technology companies into a position of global competitiveness. It can provide the benefits to industry of vertical and horizontal integration without undesirable restrictions. It may provide a uniquely U.S.-style cooperative business structure. 


\section{PRODUCT DATA STANDARDS ARE THE KEY TO CONCURRENT ENGINEERING}

The critical ingredient for the use of concurrent engineering in manufacturing is the integration of product and process data. This integration gives the designer, along with everyone else in the commercialization chain, information about the entire life cycle of a product, as well as information about how their decisions affect all other aspects of the product. In addition, the integration and automation of product and process data provides for meeting the needs of the specialists involved with a product's commercialization by allowing each of them to obtain a particular "view" of the product that is suitable for their specialty and function.

Within a single enterprise, the issue may be more one of technology than standards. Sometimes a company can choose to use systems from one vendor for all applications, or it can develop the interfaces, translators and other software needed to integrate its systems. But whenever more than one company cooperates or shares information, interoperability of systems becomes the uppermost issue, and that means standards. (Standards do not guarantee interoperability, but they bring it closer to reality.)

The integration of product and process data is not possible unless there is a mechanism that allows the sharing of information among different manufacturing systems. The mechanism must be a standard digital representation for product and process data. That is why product data standards are the key to multi-enterprise concurrent engineering and why, in today's business environment, concurrent engineering is impossible without standards.

\section{A. The Path From Automation to Concurrent Engineering}

Automation in manufacturing has led to impressive economic benefits from improvements in capacity, productivity and product quality. Yet, the benefits that remain unrealized are even greater. They are the benefits that will accrue from the integration of information among automated systems. The benefits include:

Reduced time from concept to commercialization. The efficient sharing of product data among automated systems will eliminate the need to produce hard copy drawings and models. Design details can be tested electronically against physical and engineering constraints using analysis systems and against economic constraints using cost prediction and manufacturing process simulation systems. Design changes can be made rapidly even after initial production has begun.

Reduced costs. Increased productivity will be obtained by the increased efficiency of the design process (discussed in Section III) and by reduced "time to market." Studies have shown that concurrent engineering results in a reduction in the number of design changes and in the amount of material wasted due to defects and rework [1].

Increased responsiveness to customer needs. By improving the flexibility of the bond between design and production, manufacturers can more quickly introduce new products or change existing products. This capability is essential in today's global markets. The demands by 
customers for both products and services that are characterized by differentiation, customization, and localization, result in a competitive environment where the rewards go to the speediest and quickest to adapt.

Increased cooperation among suppliers and vendors. The ability to communicate and exchange information among suppliers automatically spreads the benefits of integration from within a single enterprise to a network of enterprises. For example, a single design change in one component can cause an unpredictable delay as its effects cascade through all enterprises whose components and processes are required by the product. Today, even the need to evaluate the impact of a design change on the product causes delays as different enterprises communicate and respond. However, integration would not only allow manufacturers to coordinate activities and product changes among all their suppliers to avoid delays, but, even more importantly, it also would allow manufacturers and suppliers to take any required actions automatically and simultaneously.

The integration of information means the merging of machines and information into a system that is responsive and efficient--a system that can support concurrent engineering.

\section{A Higher Level of Automation}

Typically, many different computer-aided tools require access to computerized product data. The product data represents all the information about the product, including the product's function, its design, the reasons for its design features, and the manufacturing processes that are used to make it. Ideally, the data also describes how the product is to be used or operated, how it is to be maintained and repaired, and how it is to be properly deactivated or disposed of.

The computer-aided tools and computerized information systems that use product data are essentially very large computer programs. They were developed over many years by many people. Because they were developed independently, they tend to use unique representations for storing data. Unfortunately, each system is only able to use data that has been stored in the particular representation that it accepts.

The problem of integrating these systems is the same problem of communicating among people who speak different languages. Each time product information is transferred from one system to the next it must be translated or reformatted. Obviously, having to perform this extra step to share information is inefficient and costly. The costs multiply when many systems are involved. The number of different translators required for a number of different systems to communicate is the square of the number of systems.

The primary reason usually cited for using information systems technology is to reduce costs. Ironically, the incompatibility among existing information systems has the opposite effect: it increases costs. A solution must be found that enables the sharing of product data among different manufacturing information systems. The key to the solution is a standard for product data representation and exchange.

Achieving the goal of concurrent engineering and the economic benefits it represents, is hindered not only by existing incompatibilities but also by the complex nature of the data that must be shared 
among systems. The manufacturing data that must be shared is more than just an accumulation of unrelated bits of numerical information. Design data provides a good example; it contains physical and functional information as well as information about the significance of the design decisions that led to the final design. Product data includes not only the design data itself, but also data about its supporting infrastructure and its interfaces with other equipment.

Therefore, the standardization of product data implies more than merely the standardization of product data file formats. For concurrent engineering, information models for product data are needed. The path from automation to concurrent engineering is through standardized product data models.

Key to the goal of concurrent engineering is the ability of manufacturing information systems to capture automatically the knowledge that is generated during the product life cycle. The knowledge can then be used by the designer and others involved in managing the product. For example, knowledge about how a product would be processed or what new materials would be required to meet the functional specifications is made available to the designer as the design is being developed to ensure the best quality product reaches the downstream life cycle managers. In this way the most appropriate and cost-effective materials and processes can be used.

In a sense, two attributes of integration can elevate automation to a higher level:

1. The ability to capture automatically the knowledge gained during designing, producing, commercializing, and managing a product throughout its lifetime, and

2. The ability to exchange automatically that product knowledge among different computer systems.

An integrated level of automation can be thought of as a facilitating or an enabling technology for concurrent engineering.

\section{Technical Issues in Shared Databases}

Automated systems store product information digitally in a database. The mechanism for sharing the information is a multi-user or "shared" database. In a shared database environment, the product information can be accessed by one or more applications, even at the same time.

Of course, there are many technical issues that must be resolved for such a shared database environment to be implemented. As was already described in Section III, an interface is needed between present computer-aided design representations of a product and other computer-aided systems, such as computer-aided engineering analysis and process planning systems. Production costs and capabilities must be integrated into the database for effective design decisions.

Intelligent processes must have access to geometry data in much the same manner that users query business systems. In addition, it is critical to have a mechanism that allows new knowledge to be added to the database as the intelligent processing operations are being performed. 
The technical challenge is the development of the information technology and the associated standards that will define the environment for the representation of product knowledge. This will allow the implementation of a shared-database environment for concurrent engineering.

\section{B. The PDES/STEP Effort}

PDES, which stands for "Product Data Exchange using STEP," refers to the U.S. activities in support of the development of an international standard for product data sharing informally called STEP, the "Standard for the Exchange of Product Model Data." PDES will help establish a standard digital representation for product data. The specifications already developed by the PDES effort have been submitted to the International Organization for Standardization (ISO) as a basis for the evolving international standard STEP. As the PDES and STEP efforts share common goals, they are sometimes referred to jointly as "PDES/STEP," or simply just as "STEP."

It is important to recognize that STEP is more than a standard for the representation of product data. The development of STEP is a pioneering effort that includes the research and development of the information technology necessary for the envisioned shared-database environment. Once this standard and its environment are in place, all types of enterprise information can be more easily shared.

\section{A New Approach To Standards}

To achieve the goals of the PDES/STEP effort, a new approach to the standards-making process is required. This approach facilitates cooperative development of the requirements, the information technology, and the specifications simultaneously--before the existence of commercial systems that can use the capabilities of the standard.

The technology does not yet exist to define a product and its associated properties and characteristics completely. Even if this could be done now, the technology does not exist to communicate this information electronically and to interpret it directly by the wide variety of automated systems associated with the product's life cycle. Therefore, only a process for creating standards at the same time the technology is being created will succeed for STEP.

The creation of specifications for the standard representation of product data involves many complex issues. It requires a number of different information and manufacturing system technologies and the experience of many different kinds of technical experts. Institutional support for voluntary national and international standards organizations is provided by businesses and industrial consortia and government agencies.

There is a great need for consensus. Industry users and software vendors must cooperate closely throughout the precompetitive technology development and the standardization processes.

In addition, it is essential that the standardization process includes rigorous testing to determine that the standards meet the needs of the user communities. Testing is discussed in Section C, The Technical Challenge of STEP. 


\section{U.S. Government Needs and PDES/STEP}

In 1988, an ad hoc U.S. government interagency task group was formed to focus on information sharing among interested federal agencies. The objectives of the group were to: "prepare and consolidate government requirements for input into PDES development activities, and provide recommendations as to technical and other actions such as needed policy changes, regulatory changes or contractual vehicles/tools (e.g. data item descriptions, contract clauses, etc.) which the government should put in place to foster the development of the PDES specification" [16]. Some of the concerns about the current product data environment expressed in the task group report are:

- It is hard-copy oriented.

- It is massively heterogeneous in terms of vendors and system age.

- Product knowledge is not well captured.

- Product cycles (from R\&D to production) are very long and the handoff from one phase to the next phase often loses information.

- Technical data packages are often in error and incomplete.

- Incorporation of changes and technology upgrades is slow.

- New efforts often just automate existing methods.

- Transfer of information to and from contractors is slow.

- Funding for "non-product" development such as PDES is limited and sometimes nonexistent.

- Acquisition of improved technology (e.g., new computers and CAD/CAM/CAE) is difficult, time consuming (average 3 to 5 years), and done in the face of ever-shortening technology half lives.

- Industry concern with proprietary data rights is at odds with government desires.

- There is a reluctance for legal reasons to provide $\mathrm{CAD} / \mathrm{CAM}$ data rather than part drawings.

- Data is replicated many places for different purposes (e.g., non-common/non-integrated databases).

PDES is a major component of the U.S. Department of Defense (DOD) Computer-aided Acquisition and Logistic Support (CALS) program [17]. CALS is tackling a related, but larger scale set of issues:

- Developing and testing standards for digital technical information;

- Sponsoring the development and demonstration of new technology for the integration of technical data and processes;

- Implementing CALS standards in weapon system contracts and encouraging industry modernization and integration;

- Implementing CALS in Department of Defense information system modernization programs.

The emphasis of CALS is the sharing of information by industry and government. The philosophy is that this can only be accomplished through integrated databases that can be accessed by a variety of heterogeneous computer systems, as illustrated in Figure 6. According to CALS, STEP represents the methodology to help accomplish the goal.

Late in 1990, the Department of Commerce and the Department of Defense signed a Memorandum of Understanding (MOU) to "accelerate the development and deployment of technology that will 

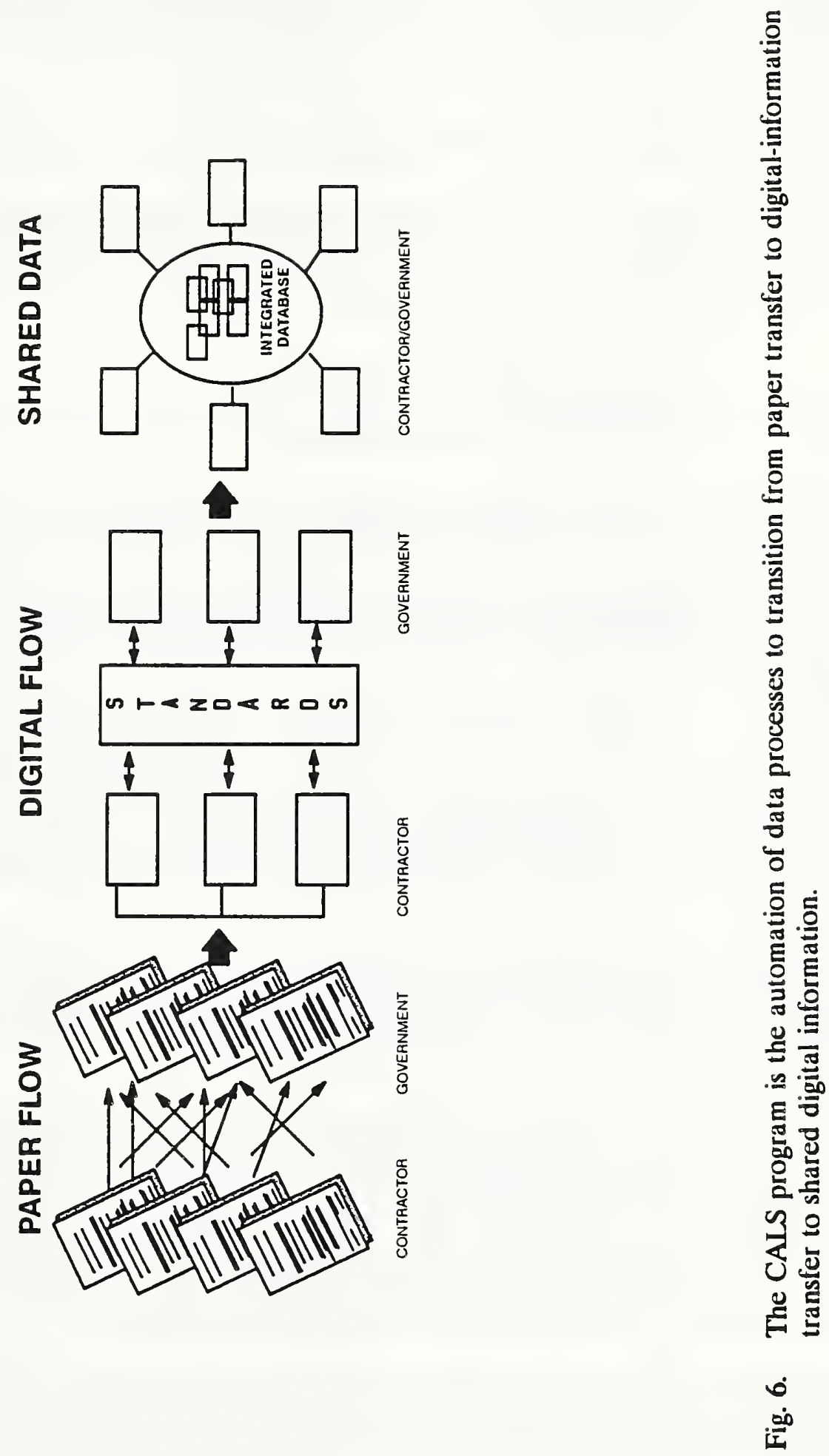
result in higher quality, shorter time to production, and lower costs for both weapons systems and commercial products." Cited as essential to this goal are the development of new technology and standards such as STEP. The MOU outlines a partnership program of development, testing, and implementation of standards for product data exchange. The Department of Energy and NASA are expected to enter into similar MOUs with the Department of Commerce in this arena.

\section{Institutional Aspects of STEP Development}

There are a number of organizations working at both the national and international levels to develop an exchange specification for product data. They include the following organizations:

- IGES/PDES Organization

- ISO TC184/SC4

- ANSI US Technical Advisory Group

- PDES, Inc.

- NIST National PDES Testbed

(The role of the National Institute of Standards and Technology [NIST] and the NIST National PDES Testbed are discussed in the following sections; the other organizations are discussed below.)

IGES/PDES Organization. The concept of PDES grew out of the Initial Graphics Exchange Specification (IGES) effort. At the time, the acronym PDES was Product Data Exchange Specification.

IGES was first published in 1980 and was updated in 1983, 1986, 1988, and 1990 [18]. Its goal is to allow CAD data to be exchanged between systems built by different manufacturers. When IGES data is passed between design systems, considerable human interpretation and manipulation of data may be required. Since IGES was designed primarily as a mechanism for file exchanges between $\mathrm{CAD}$ systems, it is not able to support shared databases between dissimilar product life cycle applications.

IGES developers recognized that a more sophisticated standard would be required to support the integration of different types of product life cycle applications. Therefore, the PDES/STEP effort focused on developing a complete model of product information that is sufficiently rich to support advanced applications, and to support concurrent engineering.

The U.S. voluntary organization that is conducting technical activities in support of the development of PDES/STEP is the IGES/PDES Organization (IPO) [19]. The IPO is chaired by the National Institute of Standards and Technology (NIST) and administered by the National Computer Graphics Association. In 1985 a formal study, called the "PDES Initiation Effort," was conducted. It established a framework and the methodologies for subsequent PDES/STEP activities. Approximately 200 technical representatives from the United States and other countries meet four times each year to address PDES/STEP-related technical issues.

ISO TCI84/SC4. In 1983 a unanimous agreement was reached within the International Organization for Standardization (ISO) on the need to create a single international standard which enables the capture of information to represent a computerized product model in a neutral form without loss 
of completeness and integrity, throughout the life cycle of a product [20]. In December of the same year, ISO initiated Technical Committee 184 (TC184) on Industrial Automation Systems. Subcommittee 4 (SC4) was formed at that time to work in the area of representation and exchange of digital product data.

Currently, twenty-five countries are involved in the work of SC4. Sixteen of these countries are participating members and nine are recognized as observers. The U.S. is a participating member. The SC4 Chair and the Secretariat are currently held by NIST.

Technical support for SC4 comes predominantly from its working groups (WGs). Alternate quarterly meetings of TC184/SC4/WG level are held concurrently with the IGES/PDES Organization quarterly meetings. Many of the same technical participants from the U.S. and other countries are active in both organizations.

In December 1988, the draft PDES Specification, developed through the voluntary activities of the IGES/PDES Organization, was submitted to SC4 as a draft proposal for the international standard STEP.

ANSI US Technical Advisory Group. The American National Standards Institute (ANSI) is the recognized U.S. representative to ISO and provides the basis for U.S. participation in the international standards activities relating to PDES [21]. To ensure that the positions on standards that are presented to ISO are representative of U.S. interests, a mechanism has been established for the development and coordination of such positions. ANSI depends on the body which develops national standards in a particular technology area to determine the U.S. position in related international standardization activities. Such bodies are designated by ANSI as "US Technical Advisory Groups" for specific ISO activities.

As a participating member in ISO TC184/SC4, the ANSI US Technical Advisory Group (US TAG) selects the U.S. delegates to SC4 and advises the delegates on how they should vote on issues presented to SC4. The US TAG usually meets at each IPO quarterly meeting.

The current US TAG to TC184/SC4 was formed in 1984. Its membership is comprised primarily of technical experts from the IGES/PDES Organization. This type of representation ensures that the technical changes that U.S. engineers and computer scientists believe are necessary are reported to ISO for consideration. ANSI has selected NIST to be the secretariat.

PDES, Inc. In April 1988, several major U.S. technology companies incorporated as PDES, Inc. with the specific goal of accelerating the development and implementation of PDES. The South Carolina Research Authority (SCRA) was awarded the host contract to provide management support. The technical participants provided by the PDES, Inc. member companies and SCRA's subcontractors are under the direction of the PDES, Inc. General Manager from SCRA. At present, there are twenty-four companies that are members, including two foreign companies. Member company's combined annual sales total over $\$ 400$ billion; they employ over three million people.

PDES, Inc. has embarked on a multi-phased plan for the acceleration of STEP development. Initially the emphasis was on testing and evaluating a data exchange implementation of mechanical parts and rigid assemblies. Current efforts focus on the identification of software implementation requirements, construction of prototypes, and development of "context-driven integrated models" for 
small mechanical parts. Recently, PDES, Inc. restructured and broadened the program scope to include such areas as electronics, sheet metal and structures. PDES, Inc. is providing increased leadership in the effort to accelerate the implementation of STEP [22]. NIST is a government associate and provides a testbed facility and technical team members to support the PDES, Inc. effort.

\section{Nature of STEP}

The many different organizations and individuals that are involved in the development of STEP share a common interest:

The establishment of a complete, unambiguous, computer definition of the physical and functional characteristics of a product throughout it's life cycle.

As a standard method for digital product definition, STEP will support communications among heterogeneous computer environments. STEP will make it easier to integrate systems that perform various product life cycle functions, such as design, manufacturing and logistics support. Automatic paperless updates of product documentation will also be possible. The principal technique for integrating these systems and exchanging data will be the shared database.

In the context of STEP, a product may range from a simple mechanical part, such as a bolt or a screw, to a complex set of systems, such as an aircraft, a ship, or an automobile. Ultimately, STEP should be able to represent the information which is needed to describe all types of products, including mechanical, electrical, structural, etc.

STEP addresses many questions about a product: What does it look like? (geometric features); How is it constructed? (materials and assembly); For what function is it intended? (structural and functional properties); How can we tell a good product from a bad one? (tolerances and quality constraints); What are its components? (bill of materials).

The STEP specification is being produced as a series of documents called "parts" [20]. Currently identified parts of the specification ${ }^{1}$ are:

- Introductory:

Part 1. Overview

- Description Methods:

Part 11. The EXPRESS Language

- Implementation Forms:

Part 21. Clear Text Encoding of the Exchange Structure

- Conformance Testing Methodology and Framework:

Part 31. General Concepts

'This list of STEP part titles is current as of February 1991. 
Part 32. Requirements on the Testing Laboratory

- Integrated Resources:

Part 41. Fundamentals of Product Description and Support

Part 42. Geometric and Topological Representation

Part 43. Representation Specialization

Part 44. Product Structure Configuration

Part 45. Materials

Part 46. Presentation

Part 47. Shape Tolerances

Part 48. Form Features

Part 49. Product Life Cycle Support

- Application Resources:

Part 101. Draughting

Part 102. Ship Structures

Part 104. Finite Element Analysis

Part 105. Kinematics

- Application Protocols:

Part 201. Explicit Draughting

Part 202. Associative Draughting

Part 203. Configuration Controlled Design

Part 204. Mechanical Design Using Boundary Representation

Part 205. Mechanical Design Using Surface Representation

The number and titles of the parts are likely to change often as new needs are identified; existing parts may be revised or additional parts may be added to the standard.

There are likely to be many additional application protocols. This is because application protocols are central both to progress in improving and completing STEP and in commercializing it. Application protocols are discussed in the next section.

STEP is defined and represented officially in the EXPRESS programming language [23]. EXPRESS was designed to represent information models in a form processible by computers. The STEP EXPRESS model, called a "conceptual schema," defines and identifies the "objects," or "entities," that can be used by STEP applications. In the first implementation form, STEP product models will be exchanged using a STEP "physical file" [24]. EXPRESS is still being modified and improved to meet the needs of STEP. It will ultimately become an ISO standard (Part 11 of STEP).

IDEF1X is a modeling language that has been used to represent STEP graphically [25]. EXPRESS$\mathrm{G}$ is a newer modeling language extension to EXPRESS. Examples of EXPRESS-G and EXPRESS representations of two simple STEP entities are shown in Figure 7. There are a variety of software tools available for processing EXPRESS [26]. 


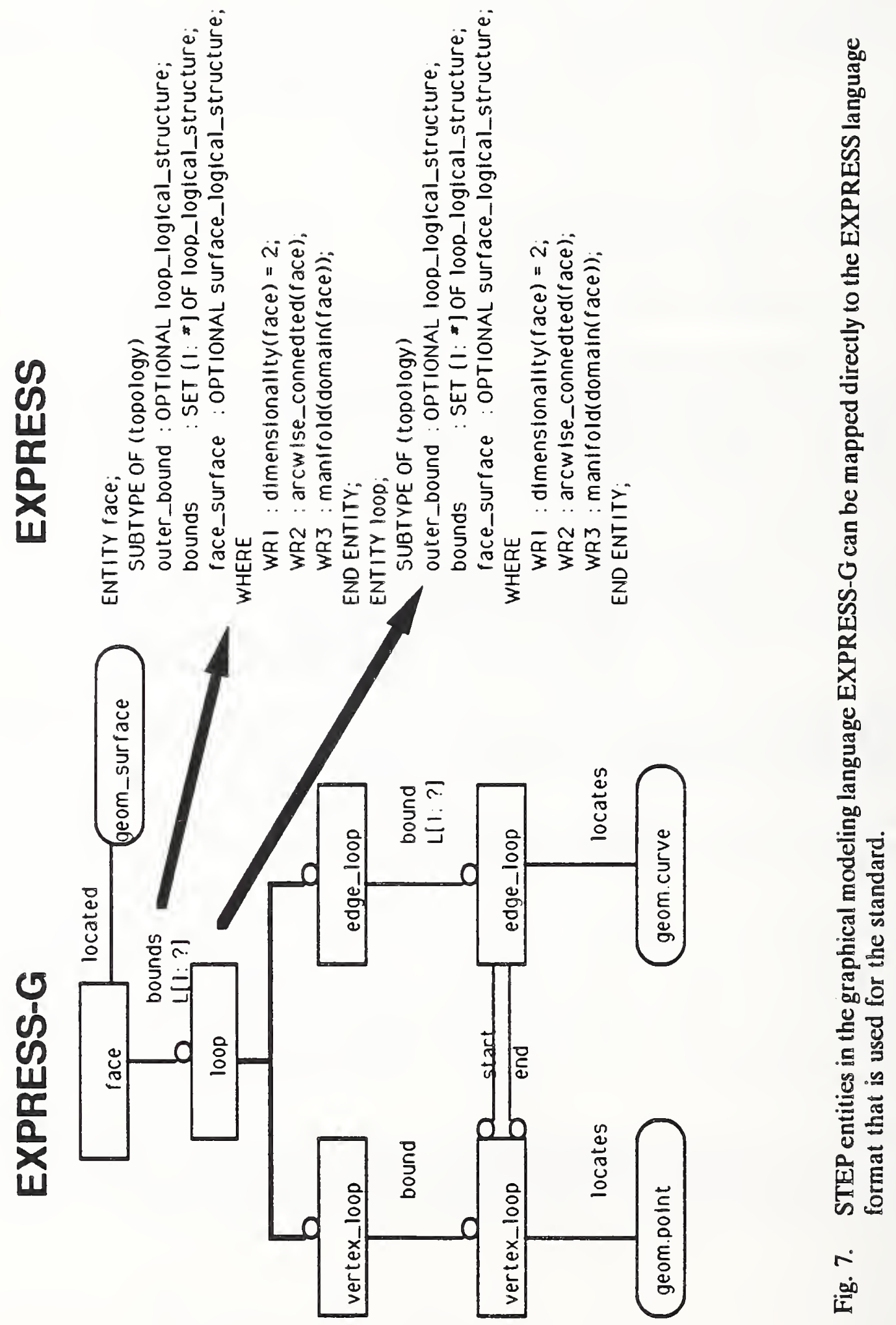




\section{Commercialization of STEP}

Representatives from industry have key roles in each of the organizations working to develop STEP. Industry must define the requirements for STEP and must assume the most critical role of implementing commercially viable STEP-based systems. After these systems are implemented, industry and government will have to coordinate their efforts to transition jointly from existing information systems to those based upon STEP.

The ultimate objective of PDES/STEP activities is the commercial availability of STEP-based systems. Commercial system developers need:

- Technical specifications that are sound and easy to implement,

- Commercially fair standards that do not favor competitors, and

- A large potential market for their products.

To ensure that STEP is a success, it is necessary that the foundation be built while the specifications are still under development. The problems and issues that will eventually be faced by system developers and users must be identified and addressed before the specifications become standards.

Vendors of systems that will use STEP need to feel confident that the standard is complete, consistent and stable before they will invest in development efforts. Vendors must have easy access to the most current versions of STEP. Help should be freely available to assist their understanding of the standard and how to implement it. Finally, vendors need to know that there is a clearly defined market for systems that employ STEP.

Vendors will use application protocols to build their products. For this reason, the strategy is to implement STEP through application protocols, and to extend STEP through the development of new application protocols that bring new entities into the standard as their need is identified.

Application protocols will be standards that define the context, the use, and the kind of product data that must be in STEP for a specific manufacturing purpose in a product's life cycle, such as design, process planning, and NC programming [27]. Application protocols standardize the use of STEP to support a particular manufacturing function reliably and consistently.

An application protocol consists of [28]:

1. An application reference model that specifies the kind of data required to perform a particular purpose, in terms that are appropriate and familiar to experts in the -application area,

2. An application interpreted model that defines how the STEP data is to be used to present the information specified in the application reference model,

3. Documentation that describes how the information is used and exchanged, and

4. A set of conformance requirements and test purposes. A corresponding abstract test suite will be developed for each application protocol. 
The commercialization of STEP is intimately tied into the development and conformance testing of application protocols.

\section{Harmonization Among Different Types of Product Standards}

Harmonization, involves the integration, or consolidation, of standards that may be overlapping or conflicting into an unambiguous set of standards that are consistent, compatible, and complementary. Harmonization represents a broad and complex challenge; it must deal with different types of both existing and emerging standards in a variety of industries. Because PDES/STEP development activities are addressing the underlying enabling technologies, PDES/STEP can contribute to harmonization of all product standards.

However, even if there were no overlap or conflict among product data standards, harmonization would still be necessary because complex products include a variety of types of components, and therefore their manufacture requires mechanical, electrical, and other types of data.

Under the auspices of the Industrial Automation Planning Panel of ANSI, an organization called the Digital Representation of Product Data Standards Harmonization Organization was formed to "facilitate the efficacious use of digital representation standards providing a forum for coordination, planning, and guidance to standards developers and approvers" [29]. The harmonization organization has as a long-term objective an integrated set of standards that can support, in digital form, the definition of products for all aspects of their life cycles.

The Organization intends initially to support efforts to integrate four standards sanctioned by ANSI that address the representation and exchange of product definition for electronic products, and to help harmonize them with STEP. These standards, used in electrical, electronic, and electromechanical design and manufacturing, have considerable overlap and conflict and are not consistent with STEP. They include:

- VHDL, "Very High Speed Integrated Circuit (VHSIC) Hardware Description Language," an algebra-like description that is used to design complex logic for chips and computers,

- EDIF, "Electronic Design Interchange Format," a file format for communicating twodimensional graphics and interconnection information that is often used to describe the patterns that are used to fabricate semiconductor chips,

- Institute for Interconnecting and Packaging Electronic Circuits (IPC) Series 350, used to describe the patterns and mechanical process to manufacture printed circuit boards, and

- IGES, "Initial Graphics Exchange Specification," used to represent the three-dimensional geometry of objects.

Companies that produce electronic products often must use all four of these standards. Unfortunately, since the standards do not work well together, the product information often must be reentered into different computers as the product progresses through its life cycle stages.

The Organization must first define the means for an integrated network of digital product data standards and the definition of a common modeling methodology for all product data standards. (In 
STEP, EXPRESS is the specification language and IDEF1X is one of several modeling tools.) Once a methodology is accepted, all product data standards models could be integrated into one model. There would also be a common glossary of terms and a dictionary of data entities.

Another aspect may be a structure or "taxonomy" that defines the interrelationships among all product technologies. Such a taxonomy could become a "roadmap" for future standards activities and extensions to existing standards.

\section{The Technical Challenge of STEP}

There are four major technical challenges facing the developers of STEP:

- The exchange of data is different from the exchange of information. Data must be transmitted accurately and without any changes. In contrast, information, although composed of data, must be understood and interpreted by the receiver. Furthermore, the receiver must be able to apply the information correctly in new situations. The first challenge is that STEP is a standard for information, not just data.

- The need for STEP to be extendable to new products, processes, and technologies, requires a more abstract representation of the information than in previous standards. Regardless of their equipment or process, a user must be able to obtain the information necessary to do something from the STEP representation of a product. Therefore, the second challenge is that the development of STEP must include the development of an "architecture" or a framework for the exchange of information, not just a means or format for storing information.

- The wide range of industries and the diversity of product information covered in STEP is beyond that of any previous digital standard. The variety of attributes and parameters, such as geometric shape, mechanical function, materials, assembly information, and date of manufacture, is immense. Also, the industrial base, the number of industries involved, is enormous; even greater is the number of technical disciplines that are involved. Moreover, STEP must be flexible and extensible so that new information and additional application protocols can be added and can be upwardly compatible. Therefore, the third challenge is that the scope and complexity of STEP is far beyond any previous standards effort.

- Traditionally, standardization is a process that devises an approach encompassing a variety of existing vendors' options, builds on the best solution available, and avoids penalizing some vendors more than others. In the case of STEP, there is no existing implementation. Thus the fourth challenge: the technology to support STEP must be developed at the same time the standard is evolving.

The consensus approach to meeting the above challenges is to start with conceptual information models [30]. STEP will consist of a set of clearly and formally defined conceptual models and a physical exchange protocol based on these models. The conceptual models will be combined into a single model with a standard interface to a shared database [31].

The following sections describe the approaches used by the community that is working to develop and implement STEP successfully. 


\section{Data Sharing}

Clearly, it is not the physical hardware connections between computers that is the major issue in data sharing; it is incompatible software. The root of the problem is proprietary data representations, that is, vendor-specific data formats. More often than not, the vendors of computer applications store the data which is required and produced by their systems in their own proprietary format.

For example, once the design of the product has been completed on a CAD system, it is stored in a data file. Some of the information in that data file represents the shape and size of the product. In an integrated information systems environment, the designer should be able to send that data file over to the manufacturing planning system. The same data would then be used by the planning system to determine manufacturing processes for the product, based in part on its specified shape and size.

If the planning system can read the contents of the design data file, it can obtain the shape and size information it needs. It might be said that these two applications are integrated. But, it is a fact today that if two commercial products are integrated, it is likely that they were developed by and purchased from the same vendor. Furthermore, it is also likely that they were intentionally designed to work together from their inception. Often, it is the case today that applications offered by the same company are not integrated.

STEP is intended to address the issue of product data sharing between different computer applications running on different computer systems within the same or different organizations. STEP will provide a standard, neutral format for product data created and shared by different applications. Neutral means that the STEP data format will not favor one particular vendor.

IGES is an example of a neutral data exchange format [18]. IGES was originally intended to provide a means for exchanging engineering drawing data between CAD systems. One problem that occurred with IGES is an outgrowth of the way vendors implement the software that is required to translate their data to and from the neutral IGES data file. Currently, a vendor's translator can create IGES data files which contain data that makes sense in the context of their system. When that same IGES data file is loaded into another vendor's system, an incomplete data translation can occur because the second vendor's translator has made a different set of assumptions about the data it is receiving.

STEP goes beyond IGES both in the breadth of its information content and in the sophistication of its information system methodologies. In addition, STEP development is including the definition of subsets of product data that are specifically required for particular usage contexts. These subsets are called application protocols.

\section{Application Protocols}

STEP application protocols address the issues of completeness and unambiguity of data transfer by specifying in advance what data should be transferred in a particular context--thereby alleviating the need for vendors to make problematic assumptions. Application protocols are those parts of STEP that are relevant to a particular data-sharing scenario [27]. 
As explained in the previous section under "Commercialization of STEP," the development of application protocols permits the incremental implementation of STEP. There will be many STEP application protocols.

The concept of an application protocol allows vendors to build an application system that can interface with STEP data in a standard manner. In a sense, an application protocol is a standardized way of implementing a portion of STEP for a specific application. It is almost like a recipe for building an application [32]. The functional components are illustrated in Figure 8 and a flow diagram of steps in the development of an application protocol are shown in Figure 9.

The development of an application protocol involves incorporating specific application requirements into STEP, then testing the application protocol for completeness, correctness, compliance, and selfconsistency. It is an iterative process [32].

Among the technical issues being resolved are:

- How application protocols will communicate with each other and share product data,

- Whether application protocols will be independent of the way in which the product data is used (for example, whether the data is shared or exchanged),

- Whether a commercial application must implement an entire application protocol or if it can utilize a subset of the application protocol, and

- How, and whether, information not already contained in STEP but needed by a new application protocol will be added to STEP.

The technical challenges involved in the development of application protocols are central to the use of STEP. Their development and implementation will determine whether STEP "can actually support complete, unambiguous exchange of product data across several application system boundaries" [32]. Application protocol development will force solutions to many of the remaining issues related to the usefulness and practicality of STEP itself.

\section{Data Representations}

At the core of the data sharing problem is data representation. STEP defines the information that describes products within different computer applications and across different enterprises. The use of computer software requires that the shared-data representations be specified. Data representation schemes must identify the data elements involved, their format, their meaning, and their relation to each other. Data representations are formally defined within STEP specifications.

For example, in the geometry portion of the STEP specification, a simple data element may be called "point." The data representation for "point" might consist of three aspects: the point's $\mathrm{X}$ coordinate, its $\mathrm{Y}$ coordinate, and its $\mathrm{Z}$ coordinate. To complete the data representation, the type of numbers allowed for the point's $\mathrm{X}, \mathrm{Y}$, and $\mathrm{Z}$ coordinates must be explicitly stated. In this case they would be "real" numbers, not integers or whole numbers. Having defined the data representation for "point," other more complex data elements can also be defined that make use of the "point" data element. 


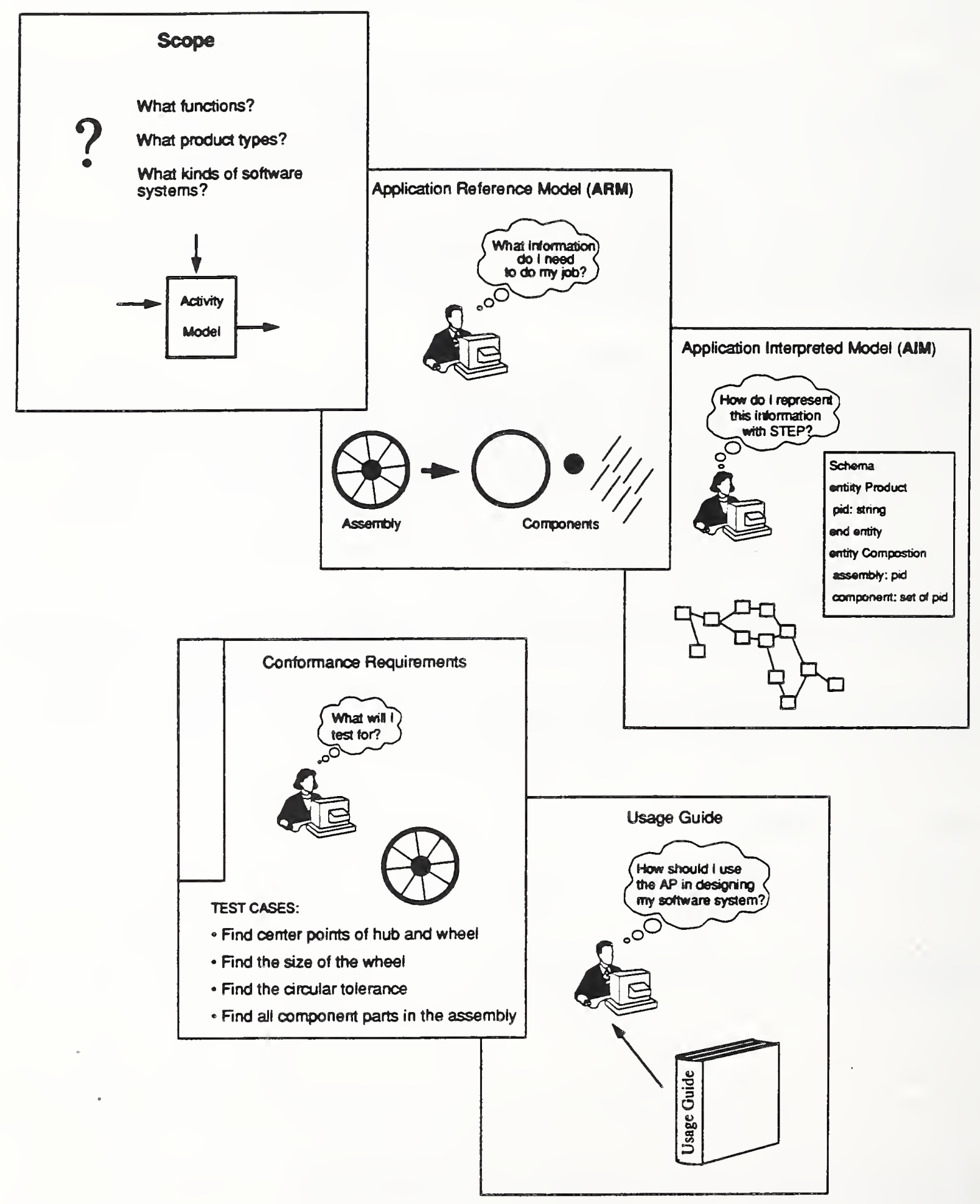

Fig. 8. The five components of an application protocol are the scope, the ARM, the AIM, the conformance requirements, and the usage guide. 


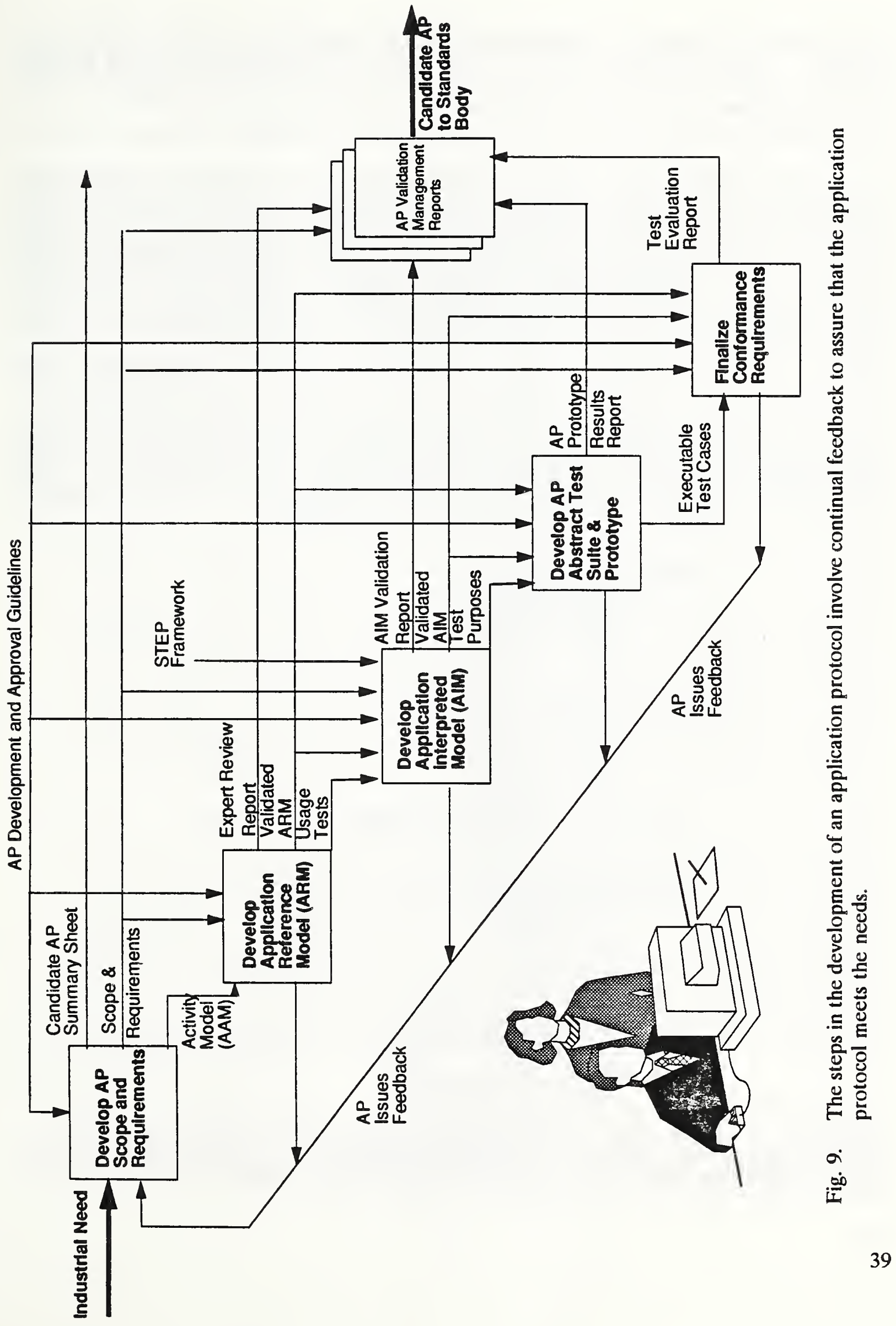


Representations for data elements can become quite complex, making them difficult to define and understand. The most important criterion for the data representations used in STEP is that they must be unambiguous. This prevents their being misinterpreted by applications, or being interpreted differently by different applications. Ambiguous data representations lead to problems like wires being mistaken for conduits, or bolts being mistaken for machine screws.

The developers of STEP employ information-modeling techniques to ensure that STEP will be unambiguous. An information modeling language is actually used to define portions of the STEP specification. Implementations of STEP are written in EXPRESS [23]. EXPRESS has many features of a computer programming language. Writing STEP in EXPRESS allows information modeling experts to use specialized computer software to check the integrity, validity, and efficiency of STEP. Besides facilitating the development of the standard itself, these information modeling techniques will also help to speed the development of future software applications based upon STEP.

STEP is organized into a framework composed of application information models, resource data models, and generic data models [33]. The generic data models, which integrate the resource data models, are the Generic Product Data Model and the Generic Enterprise Data Model [34]. The Generic Product Data Model (GPDM) contains information common to all products and meets the needs of application protocols by providing for the interpretation of generic facts in specific contexts [27]. The GPDM consists of the schemas: context, product definition, property definition, and shape representation. Currently, the definitions of the schemas, in EXPRESS, are:

$$
\begin{aligned}
& \text { gpdm_context_schema } \\
& \text { application_protocol } \\
& \text { product_context } \\
& \text { product_definition_context } \\
& \text { gpdm_product_definition_schema } \\
& \text { product } \\
& \text { product_category } \\
& \text { product_version } \\
& \text { product_definition } \\
& \text { product_definition_equivalence } \\
& \text { product_definition_relationship } \\
& \text { gpdm_property_definition_schema } \\
& \text { product_material } \\
& \text { product_shape } \\
& \text { shape_aspect } \\
& \text { surface_finish } \\
& \text { shape_representation_schema } \\
& \text { shape_model } \\
& \text { shape_model_composition } \\
& \text { shape_model_representation }
\end{aligned}
$$

The STEP data-sharing architecture must be able to access the data wherever and however it is stored. The data will be in a form dictated by the STEP Generic Product Data Model. A STEP 
data access interface may be the method used to provide application systems with the STEP data needed to perform an application [22].

\section{Technical Evolution of STEP}

A sound technical specification for STEP must address many issues pertaining to the architectures of information systems and the management of product life cycle data. Many different technologies have been brought together to establish a technical foundation for STEP. Computer-aided design and solid modeling systems provided the initial framework for describing product data. The fields of information modeling, relational and object-oriented database management systems have provided software tools that have contributed to the development effort. Technical experts who are familiar with the data requirements of design, process engineering, machine programming, and product support systems have helped define the types of data that must be supported in a product data exchange specification.

Because of the broad range of product types and application technologies which must be covered, the transformation of STEP from an abstract concept to a commercial reality is an evolutionary process. STEP application areas range from simple mechanical parts to complex electronics systems to buildings and ships. STEP is undergoing four stages of evolution:

Stage 1: Establishment of the foundation for STEP. The creation of a specification for the standard representation of product data involves many complex issues. It is virtually impossible for one individual or even a small group of individuals to write this kind of specification. The development of this specification requires both a strong technical and institutional foundation. The technical foundation for STEP is based upon a number of different information and manufacturing systems technologies and the experience of many technical experts. The institutional foundation is provided by voluntary technical activities, national and international standards organizations, businesses and industrial consortia, and government agencies. Because of the great need for consensus, all of these institutions must be in general agreement about the content of STEP, if it is going to be an effective standard.

Stage 2: Validation and standardization of technical specifications. Once an initial specification has been created, it must be validated, that is, tested to determine that it meets the needs of the user community. Validation testing takes into account how the specification will be used. Technical experts define the requirements for the different kinds of software applications that will use STEP and build information models based on the proposed STEP standards. These information models are then tested to determine whether they will meet the needs of state-of-the-art software applications. Test criteria, test procedures, and test data are also developed as part of the validation process. Only after satisfactory test results are achieved can the specification be considered workable and complete. The results and recommendations generated by validation testing flow back to the standards organizations for review and action.

Stage 3: Development of tools and prototype applications. The development of commercial STEP-based software products can be accelerated by prototyping. The developers of these prototype systems will learn a lot about using STEP technology that will help to accelerate the development of commercial products. The software tools that are developed may also 
be used in future products. If this work is done in the public domain, many companies can benefit from the results of this effort. Furthermore, early prototype applications can be used to validate the suitability of proposed standards. They can also be used for integration testing, that is, testing to determine whether or not different types of applications can work together. Prototype systems also may be extended to exercise conformance testing systems. In the absence of these prototype implementations, vendors and customers may make claims of conformance through self-testing.

Stage 4. Commercialization of and transition to STEP-based systems. Ultimately, STEP-based systems must be developed and marketed commercially. It will take a number of years for industry to recognize all of the different specialty niches for these systems and to develop stable products. Certainly this phenomenon can be seen in the personal computer market. Although the basic interface specifications for PCs were established in the early 1980's, new types of hardware and software products are still being defined today. It will undoubtedly take a number of years after products become available until they are put into widespread use within industry and government. Considerable advanced planning and investment of resources will be required to transform large government and industrial organizations into new STEP-based systems. Translation planning is essential to implementation and acceptance of STEP.

The first stage of STEP evolution is well underway, but the second stage has just barely started. Stages 2 through 4 will also have to be repeated for the different product technologies that STEP must cover, such as mechanical assemblies, sheet metal parts, structural systems, and electronics components.

\section{Verification and Validation}

Verification and validation are two ways in which commercialization of STEP-based products can be expedited. Verification is the review of both the system requirements to ensure that the right problem is being solved and the system design to see that it meets those requirements. Validation is the test and evaluation of the integrated system to determine compliance with the functional, performance and interface requirements that were verified. Validation and verification are necessary during the development of STEP and its associated software tools, as well as for the development of STEP-based products, that is, STEP implementations.

With respect to the development of STEP, validation requires testing to confirm that the requirements for the product life cycle data have been met. One of the major goals of the validation testing efforts is to test the suitability of the proposed STEP standard for product life cycle information systems applications.

Validation testing is aimed at evaluating the completeness and the integrity of the STEP specifications. Without validation testing, many deficiencies in the specifications might not be discovered until commercial applications are constructed. It is obvious that without this testing, developers might have had to bear the burden of excessive redevelopment costs and delays while the specifications are "fixed."

Validation testing is discussed more thoroughly in Section E, The NIST National PDES Testbed. 


\section{Application Systems}

Application systems are the computer software systems that will use STEP. They are systems for computer-based manufacturing functions such as computer-aided design, analysis, manufacturing planning, resource allocation and scheduling, manufacturing equipment programming, and quality assurance. Many of these systems have common data requirements and they need to share data. (A simple example of shared data is the name of the product and the identifiers of its component parts.) The early development of prototype STEP application systems is the key way to accelerate commercialization of STEP.

Some product data requirements may be unique to a specific type of application. For example, the tolerances on a product's dimensions would be required by manufacturing planning systems, but this same data would be irrelevant to scheduling systems. Yet both systems would refer to the same names when identifying the product and its components.

Ensuring that STEP addresses the requirements for manufacturing applications is a significant challenge. (This was discussed in the context of application protocols.) Generally, there are no formal, publicly available specifications of the information requirements for any of these systems. Functional requirements and design specifications must be developed for systems that will use STEP. These specifications should be defined concurrently with the evolving application protocols. They will help to determine exactly how STEP will be used by future commercial systems.

Prototype application systems should be developed that can be used to test the viability of the application protocols. Different types of prototype systems should be tested with each other to ensure that STEP permits interoperability between various applications. If the prototypes are constructed in the public domain, they can later be used as foundations and building blocks for commercial implementations.

\section{Configuration Management}

The process of developing an information processing standard involves the creation and management of thousands of documents and computer programs. Knowing which documents and computer programs are current and which are obsolete is critical to the development process. Configuration management provides the fundamental operational capability for tracking and maintaining versions of documents and software.

Configuration is the logical grouping and/or collection of elements into a coherent unit. This unit is typically a version of a software release or text document. If the configuration of an information unit is to be controlled, access and changes to the information must be controlled. Often "master" documents and approval mechanisms are established to ensure the quality and integrity of the information that is being managed.

The complexity of the configuration management problem is governed by the type of information involved and how it is to be controlled. In the case of simple configuration control systems, for example those that deal with software source code control, simple text files are usually just grouped together into a named or numbered unit and distributed as a single item. This is a simple process 
and many software products currently perform just this function. The complexity of the problem increases when the configuration involves more than just simple text files. Two examples of more complicated configuration control problems are the management of computer programs which run on different computer systems, and documents which include graphic images.

Clearly, the development of STEP is a complex configuration management problem. It involves a number of different organizations that have different interests in the technical aspects and in the status of the proposed standard. Each organization must be able to retrieve proper versions of the developing standard. Software tools are needed which can be used to merge electronic versions of text and produce a single unified document from each organization's contributions. This assembly process is one of the main functions of a good configuration management system. Reliable, controlled, and up-to-date access to an individual organization's data plus the capability to pull disparate pieces of information together is a major challenge.

The discussion of configuration management is continued in Section E., The NIST National PDES Testbed.

\section{Conformance Testing}

Before commercially developed systems are marketed, conformance testing procedures must be established which act as quality assurance mechanisms to protect both system developers and users. Conformance testing is the evaluation process or methodology that is used to assess whether products adhere to standards or technical specifications. If independent conformance testing mechanisms are not established, customers will have to accept vendor assurances that their systems comply with STEP. Unfortunately, many vendors may be incapable of determining whether or not their products faithfully comply with the standard.

The development of conformance testing methods and the development of application protocols are intertwined. Commercial systems based upon one or more application protocols will be the first implementations of STEP. Conformance testing methods for STEP will only be based on evaluating implementations of application protocols.

\section{The Role of NIST: An Engineering Paradigm}

Research and hands-on experience are essential for NIST scientists and engineers to make informed and impartial standards recommendations. Recognizing the importance of manufacturing interface standards, NIST established the Automated Manufacturing Research Facility (AMRF) in 1980 to investigate critical issues in factory automation standards. The first major goal of the AMRF involved the construction of a flexible manufacturing system, a testbed, for the small-batch manufacturing environment.

The facility is used as a laboratory by government, industry, and academic researchers to develop, test and evaluate potential interface standards. To ensure that the interface standards issue is addressed, the testbed is designed to contain component modules from a variety of vendors [35]. 
The AMRF represents a fresh approach to factory automation. The generic factory architecture incorporates elements such as hierarchical facility control, distributed database management, communication network protocols, on-line process control (deterministic metrology), data-driven (and feature-driven) processes, and manufacturing data preparation (such as design, process planning, and off-line equipment programming).

It is this experience in building a large-scale testbed facility, working with industry and universities, studying standards issues, and implementing testbed solutions that brings NIST to an important role in the development and implementation of PDES/STEP.

\section{Components of the NIST Engineering Paradigm}

Traditionally, engineering projects have been carried out by starting with specifications, developing or adapting technology, and developing the required application. Today, the management of information--especially information in electronic form--has become a critical component of any engineering endeavor. This is especially true in the work of the NIST Factory Automation Systems Division, where much of the PDES/STEP work at NIST is done. The paradigm can be used as a model for understanding and planning engineering projects.

The paradigm consists of four components:

- System Specification,

- Information Management Technology,

- Engineering Technology, and

- Engineering Application.

The paradigm is shown diagrammatically in Figure 10. The system specification component takes an industrial need such as "manufacturing world-class products" and develops the information and functional models that address the needs. The information management technology component takes the standards, in this case product and manufacturing data standards, and generates the information framework or architectecture concepts required to implement an engineering application. The engineering technology component takes the functional requirements for the applications as determined by the system specification and creates the engineering framework or architecture concepts required to implement the engineering application. Finally, the engineering application component is the integration of the two technology components into a prototype application environment to test fully the proposed set of standards. The experience gained in the application environment is used to strengthen the system specification component. The outputs are indicated by the double-lined arrows: a set of standards from the system specification component and products from the engineering application component.

The combination of the need for advances in concurrent engineering technologies and the need to represent engineering data in a standard format--STEP--is a perfect industrial problem to be implemented using the engineering paradigm. This is illustrated in Figure 11 and by the following discussion. 


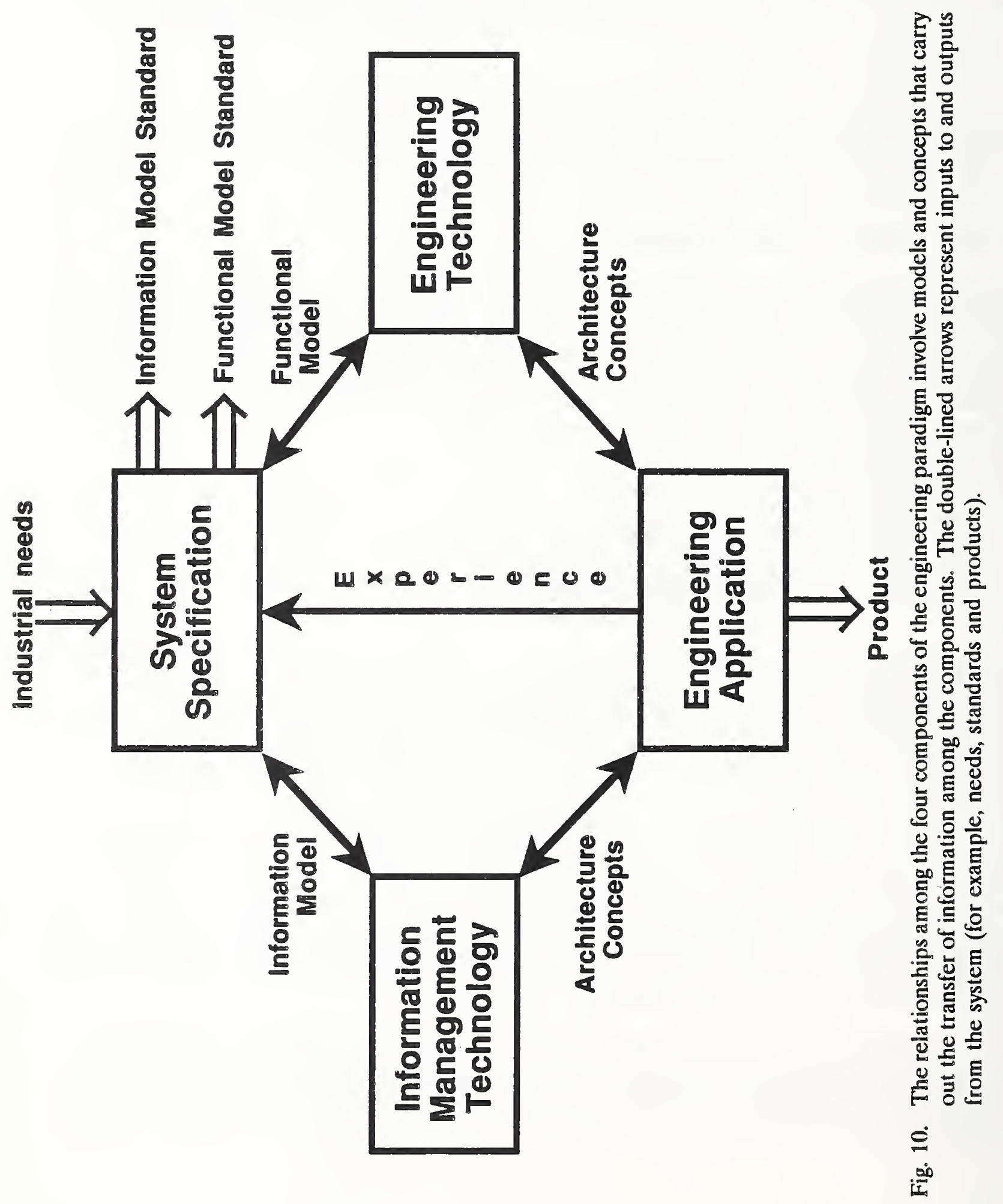




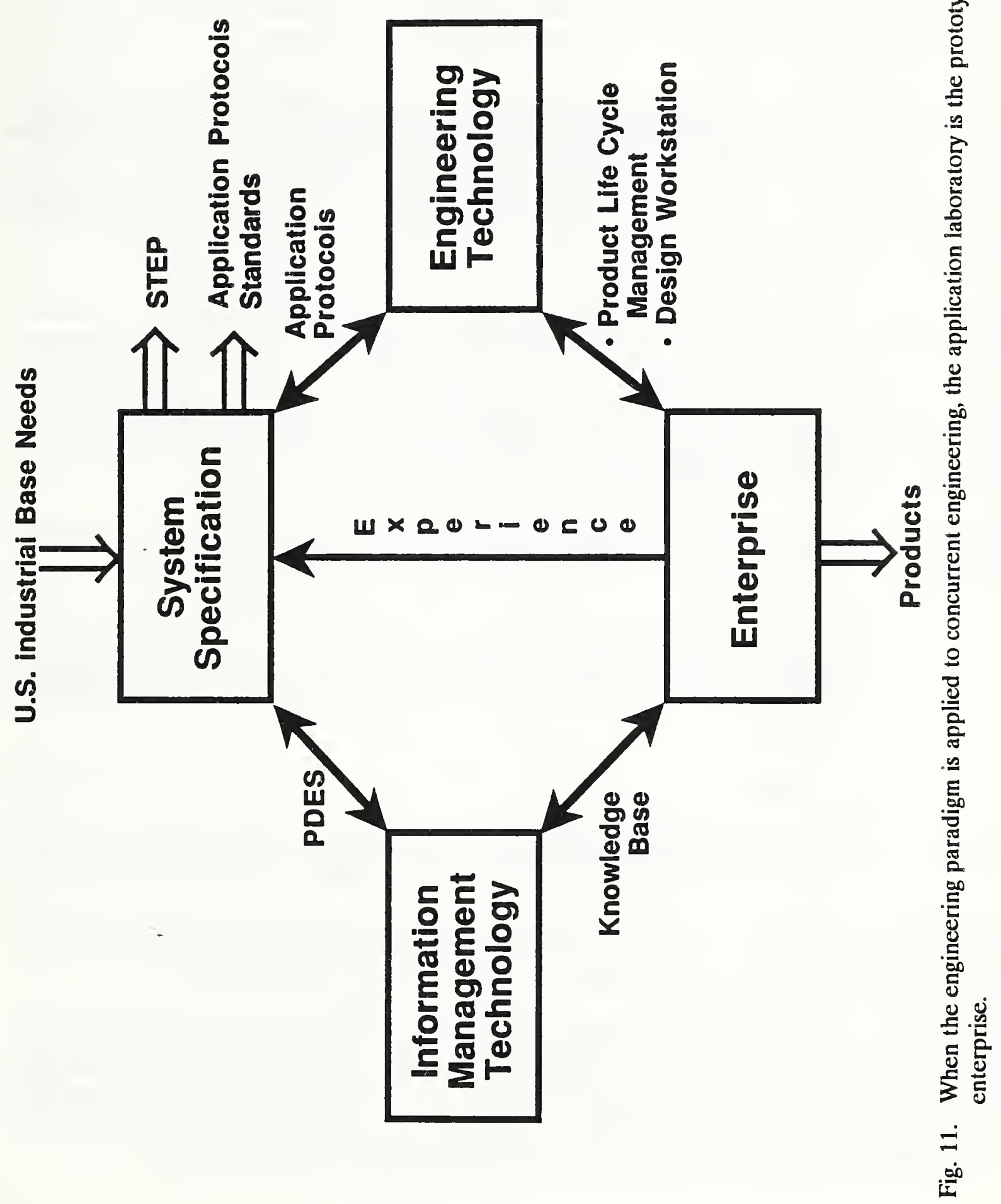




\section{System Specification}

The function of the first paradigm component, system specification, is to take industrial needs and develop the information and functional specifications required to solve them. These specifications become the basis for the development of the information and engineering technologies required to implement a solution to the industrial needs. Most of the activities performed in this component are involved with the voluntary national and international standards programs.

Therefore, in the paradigm as applied to product data-driven engineering, system specification is the development of the STEP standard as an ISO data exchange standard and the implementation of application protocols that specify the engineering environment in which STEP is to be used. NIST participates in both the formal standards organization and in the research and development of testing procedures for STEP. A staff member serves as chair of the volunteer IGES/PDES Organization. Staff also actively participate in the technical committees within the IGES/PDES Organization where robust information models that define the scope and application of STEP are developed.

NIST scientists are involved in applying to STEP the Information Resource Dictionary System (IRDS) standard being developed by ANSI [36]. There is a project that addresses the application of IRDS to STEP, including using an IRDS extendibility feature to support the storage and management of the diverse conceptual and data models of STEP. In addition, work is going on to extend the STEP information resource dictionary schema to support a full three-schema architecture, to interface STEP IRDS to software such as conceptual modeling tools and database management systems, and to develop relationships to physical design for STEP.

NIST staff are involved also in identifying the application of geometric modeling to the definition of STEP and its application implementations. There are many technical issues, such as:

- Interaction between different modeling geometry systems. As an example, for NURBS (Non Uniform Rational B-Spline) surfaces, what is the best transformation from a 5 th-order curve to a series of 3rd-order curves. In general, how is geometric information exchanged between constructive solid geometry, boundary, and wireframe models.

- Topology and its relationships to geometry.

- Geometry and topology and their relationship to application areas such as numerical control $(N / C)$ coding, graphics display, collision detection, etc.

An overriding issue is the problem of deciding what type of geometric modeler is appropriate for a given application. Research into how to categorize modeler parameters and measure expected performance for applications such as inspection and N/C coding is also important.

NIST staff participate in the development of testing procedures for STEP as well as STEP-based industrial products. They have developed test plans that identify the approaches, methodology, resources, and tasks required to test and validate STEP. There are many common testing methods that will be explored including, test data file, syntax analysis, semantic analysis, instance tables, and ad hoc database queries. 
Testing of the specification and implementations are performed at the National PDES Testbed, described in the next section. The testbed will become a model for a network of future testbeds that will be established throughout the world. The Testbed will also serve as a model for the type of software and hardware configurations and personnel resources needed to test and implement STEP.

\section{Information Management Technology}

The function of the information management technology component is to develop the proper technology to process the information identified in the system specification. The important point to be stressed is that the technology (file system or relational database, for example) must be appropriate to meet the needs of the engineering technology. The following are typical tasks to be performed:

- Determine from the information model specification the types of data representations required. Areas of concern include the implementation of a data dictionary, the types of schemas for representing the informational relationships, and the extent to which knowledge (rather than just information) needs to be represented.

- Based on the engineering environment, such as flexible manufacturing system or robot control, determine the important characteristics of the information management technology needed. The issues may include, for example, distributed vs. central storage, homogeneous vs. heterogeneous computing, version control (or configuration management), time constraints, database size, and security.

- Design and implement an information management system capable of handling the required characteristics.

In short, this component of the engineering paradigm is concerned with the conversion of STEP into an information management system that can support the engineering requirements.

\section{Engineering Technology}

The function of the engineering technology component of the paradigm is to convert the functional specifications into a collection of engineering concepts and a systems architecture that can address the industrial problem. The following are typical tasks to be performed:

- Define a plan for developing the technology. This includes decomposing the overall problem into a series of tasks and specifications.

- Identify the product data requirements and measurement systems needed. Define the control architecture and process interfaces. Develop new engineering concepts to address the problem.

- Design the overall system, including the information and functional models. Define the data requirements and the means by which data is to be collected and analyzed. 
- Determine which processes are needed for the given application, for example, process planning or robot handling.

\section{Engineering Application}

The fourth component, engineering application, is the prototyping of the concepts and architecture defined in the two components, information management technology and engineering technology. The outputs of the engineering application component are feasibility demonstrations of how the engineering and information management concepts result in a credible solution. The following are typical tasks that are performed:

- Based on the systems specification and the technology to be developed, specify the product mix to be used in the laboratory.

- Develop the interfaces between the information management systems and the engineering processes. Develop the interfaces between the various processes that compose the engineering application.

- Build a laboratory based on the architecture and concepts defined for the information and engineering technologies. Design and perform experiments that provide the proofof-concept for the technologies.

The engineering application paradigm component is realized as a laboratory in which the architecture and concepts developed in the information management and engineering technologies components are implemented. Facilities that represent processes that are part of the product life cycle are built and experiments are conducted to test the technology concepts.

At present, the AMRF can be viewed as the engineering application for the subset of the product life cycle that addresses the design, manufacturing and inspection processes. The work in manufacturing data preparation, process control, and factory control addresses the engineering technologies for flexible manufacturing. There are also efforts in data management and network communications that address the information management technology issues.

The factory control system for the manufacturing and inspection of parts uses a STEP-like format. The systems are all data driven. In fact, the vertical workstation is driven from an off-line programming environment that starts from a set of machinable features for a part [37]. The inspection workstation is driven from an off-line programming environment that generates a CAD database of the part with respect to the necessary tolerance information [38]. The five-level control architecture developed within the AMRF has become a model for the implementation of manufacturing systems.

A common thread throughout the AMRF is the standardized method of handling data. This is particularly true in the manufacturing data preparation research which is aimed at a seamless architecture based upon plug-compatible modules that streamline the preparation of data for automated manufacturing systems. 
In the AMRF, incoming part descriptions are converted to AMRF Part Model Files using commercial CAD systems and software developed for the AMRF [39]. The AMRF Part Model File includes 3-D geometric and topological information, tolerances, and other data on the part in a uniform format that can be used by other AMRF systems. Translators have been written to convert this format to STEP.

Working from the STEP files, and other information in the database system, operators then prepare "process plans" for the part. In the AMRF, these computerized plans include the cell's "routing slip," which is used to schedule the movement of materials and the assignment of workstations; the workstation "operation sheets," which detail the necessary tools, materials, fixtures, and sequences of events; and the machine tool's "instruction set," which guides the tool through the motions required to shape the part. Research is being conducted into the development and testing of a single set of standard data formats for process planning at every level of the factory control hierarchy, and an editing system to generate, archive and update these plans. In effect, the AMRF provides a laboratory for a STEP implementation. The AMRF approach to handling data is to allow the users freedom to select computers and database software, yet still be able to build an "integrated" system.

Ideally, a factory control or planning system should be able to request the information it needs without knowing which of several databases holds the information, or what format is used to store the data. A distributed database management system called the Integrated Manufacturing Data Administration System (IMDAS) is used in the AMRF to meet this need [40].

The AMRF data communications system allows computer processes such as control programs to run on many different computers and to be developed using different languages and operating systems. This system uses a method of transferring information through the use of computer "Mailboxes," which are areas of shared memory on various computers to which all machines have access through the network communications system [41].

The Manufacturing Systems Integration Program uses the AMRF as a testbed to study data and interface requirements for commercial manufacturing engineering software systems. The concentration is on functions performed during the manufacturing of mechanical parts, such as process planning, engineering design, tool management and off-line programming, shown schematically in Figure 12. The goals include demonstrating feasibility and testing integration and interface concepts for information standards to integrate manufacturing engineering and production systems.

In essence, the AMRF is the laboratory where control and metrology concepts and architectures for integrating information and technologies are implemented and tested.

Other laboratories in the Factory Automation Systems Division fulfill the paradigm expectations and perform a function similar to the AMRF for specific application areas. They include the Engineering Design Laboratory [42], which is used to evaluate software tools for integrating design and analysis and for modeling design intent and design knowledge for access and use throughout the life cycle of a product. Another example is the Apparel Design Research System, which is used to help develop methods for product data exchange that are appropriate to the apparel industry [43]. (The design project is funded in part by the Defense Advanced Research Projects Agency and the apparel project is funded by the Defense Logistics Agency.) 

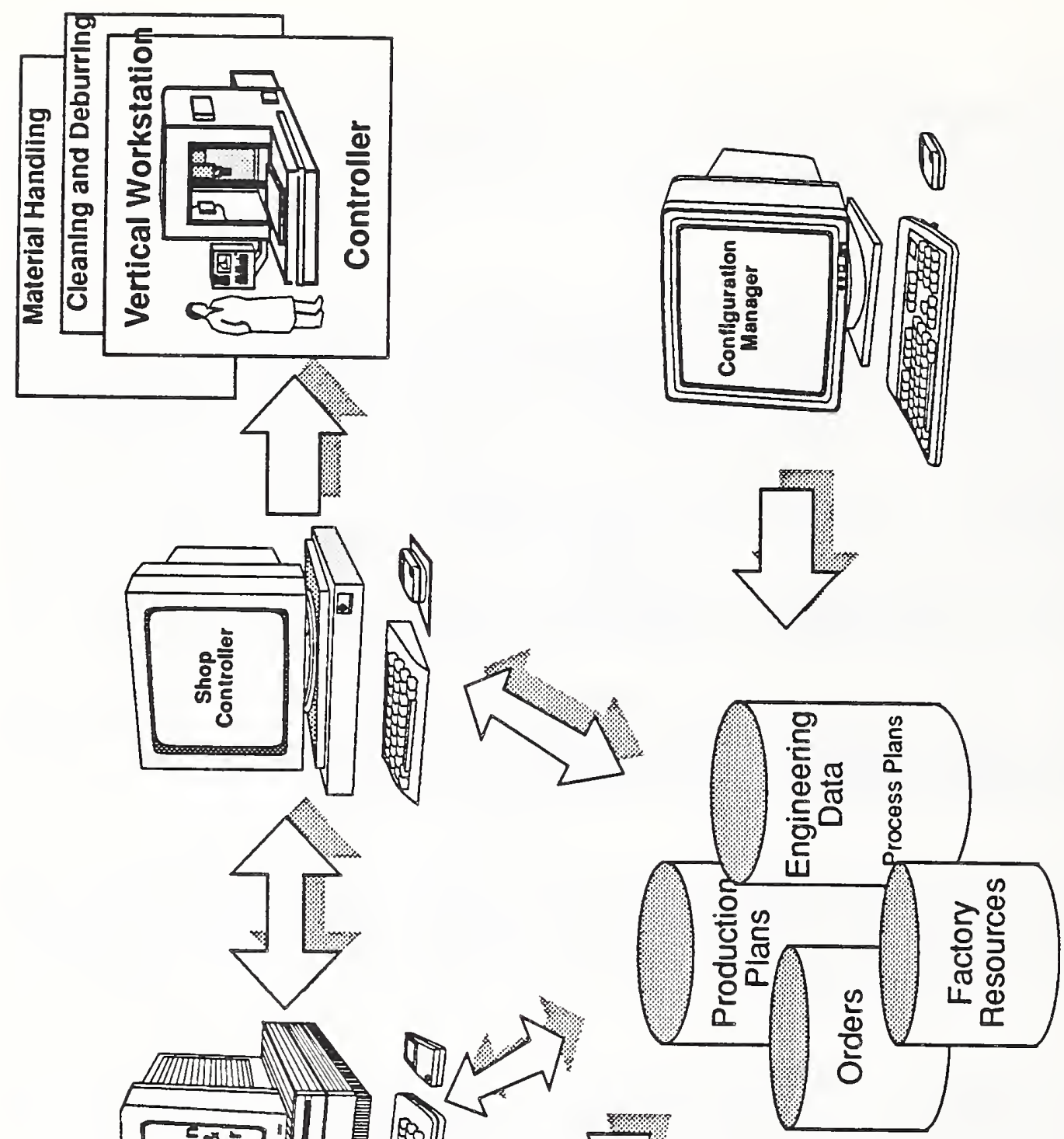

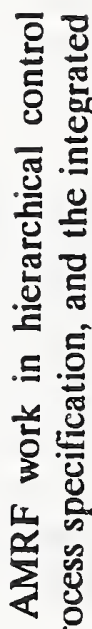

옹 흠

일

동 ్ㅐㅇ

5

흘

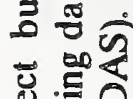

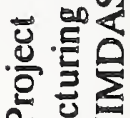

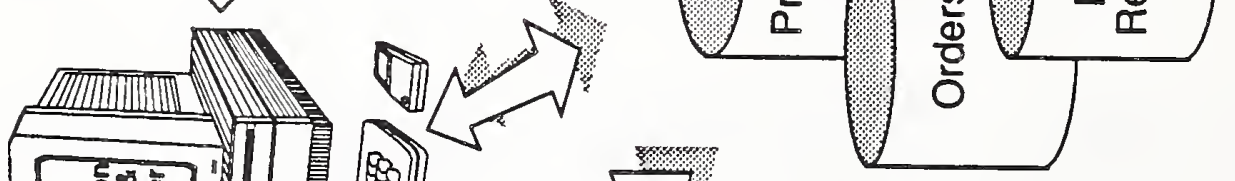

․․ㄹ

을 틀
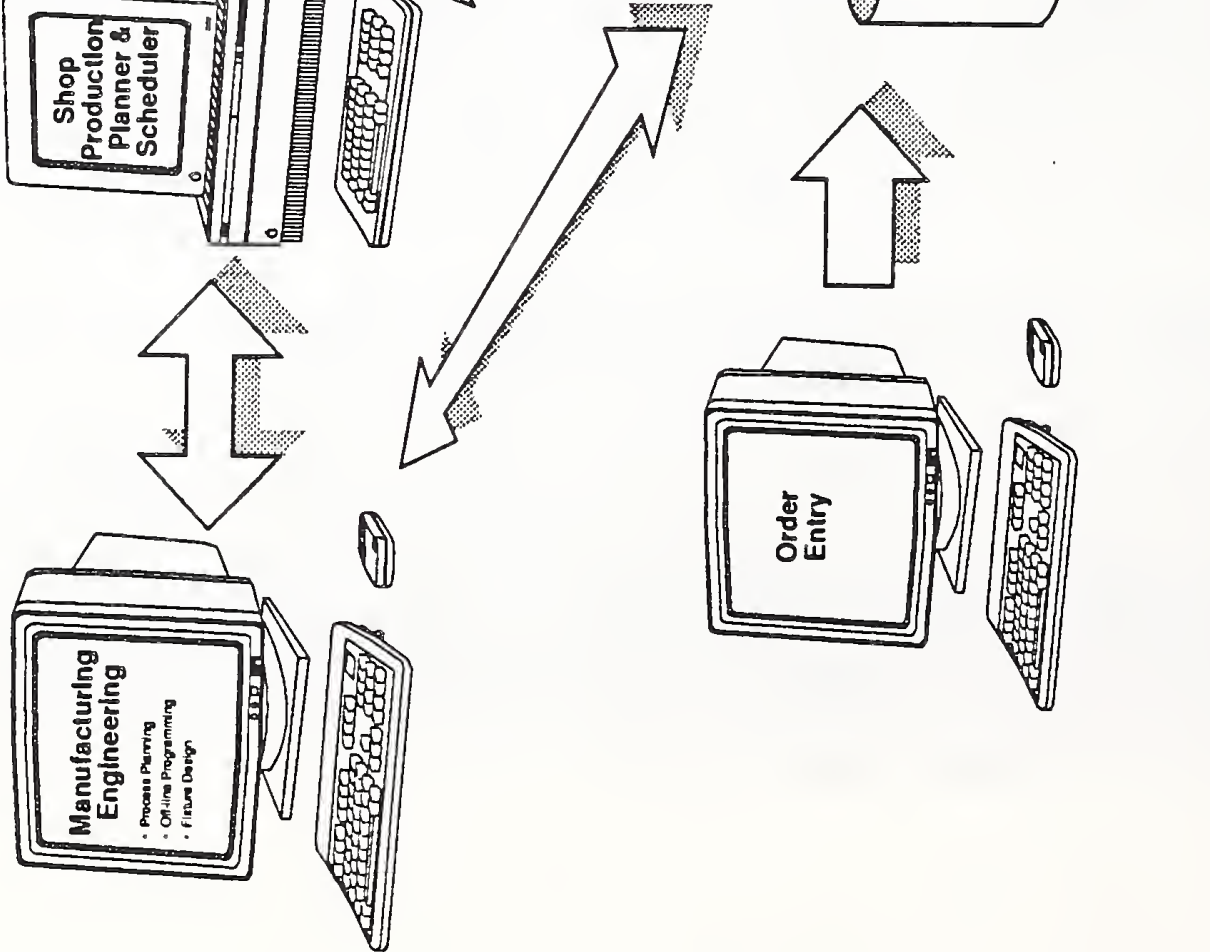

e

ए人

트 등ํำ

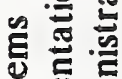

菆总

野

들

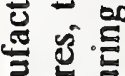

톨

$\Sigma$

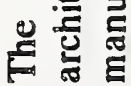

I

in 
The NIST National PDES Testbed is a focus for planning, coordination, and technical guidance of a national effort for STEP development and implementation. The national effort consists of a growing network of participating organizations of various types.

Located at the National Institute of Standards and Technology, the Testbed is a publicly accessible facility where the STEP specification and STEP-related tools can be modeled, analyzed, prototyped, implemented, and tested [44]. Physically, the facility is comprised of laboratories, computer hardware and software systems, and testing tools. The laboratories include unique laboratories such as the Validation Testing System, as well as multipurpose laboratories such as the AMRF and the Engineering Design Laboratory. The Testbed is used and staffed by leading experts on PDES issues from industry, academia, and government. It is currently staffed with the full-time equivalent of approximately 20 scientists, engineers, and support personnel.

The National PDES Testbed supports the goals of the IPO and ISO to establish an international standard for product data sharing. The Testbed was established at NIST in 1988 under U.S. Department of Defense Computer-aided Acquisition and Logistic Support (CALS) program funding. Standards which support product data sharing are recognized as a major building block in the CALS program. Under CALS sponsorship, the National PDES Testbed is advancing the development of product-sharing technologies. The staff of the National PDES Testbed are not only involved with the ISO and IPO, but also actively participate in the technical activities of PDES, Inc.

The Testbed is also the cornerstone of the Manufacturing Data Interface Standards Program at NIST. The goal of this program is the development of national standards for a "paperless" manufacturing and logistic support system.

The overall objective of the Testbed is:

To provide technical leadership and a testing-based foundation for the rapid and complete development of the STEP specification.

The major functions of the Testbed include:

Standards validation test development to ensure that the specifications and underlying information models meet the needs of product life cycle systems;

STEP application prototyping and interoperability testing to provide test cases, tools for generating test cases, and application experts who can critically evaluate the draft specifications; to ensure that the specifications are sufficiently integrated to guarantee interoperability of different types of STEP applications; and to demonstrate the advantages and suitability of STEP for use in industrial environments;

Product data exchange network integration to provide a national network at government and industry manufacturing sites and laboratories to share information and test cases; and 
Configuration management to implement a configuration management system and establish a central repository for documents and software generated by various organizations involved in the STEP development process.

\section{Standards Validation and Conformance Testing}

Validation testing is the process that ensures that STEP is usable and functional. It confirms that the standard is complete, unambiguous, and consistent. It determines that the standard meets the needs of the user community. The results and recommendations generated by validation testing must be fed back to the standards organizations for review and action. Standards committee members may then amend the specifications, affected portions may be re-tested, and the specifications can be approved as standards.

The emphasis on validation at the Testbed is on the development of computer-assisted tools for testing and evaluating proposed application protocol specifications [28].

The validation process is evolving along with STEP itself. Technical challenges still remain, including such issues as the degree of functionality that must be defined in an application protocol and that must be achieved by application systems.

To support validation testing, the Testbed provides an integrated computing environment. In addition, it acts as a repository for proof of the qualities that the STEP specification exhibits. This proof, in the form of test results and real-world test product data, will help the standardization process to proceed and will encourage implementations of information systems which use STEP.

The Validation Testing System within the Testbed is comprised of software that will: 1) automate the evaluation of the computable qualities, such as whether or not the syntax of the specification language was followed, and 2) assist validation teams with solving intuitive problems which are not feasible to automate. The names of the major component modules of the validation testing system are:

- Model Scoping and Construction Tool

- Test Definition Tool

- Test Case Data Generation Tool

- Test Case Execution and Evaluation Tool

Figure 13 illustrates the major validation testing tools and their functions.

Just as validation testing is essential to the development of STEP, conformance testing is essential to its successful implementation. Conformance testing is the testing of a candidate product's behavior and capabilities. The behavior and capabilities of the product must be those required by the standard itself, and they must be exactly what is claimed by the manufacturer of the product.

Conformance testing helps to assure product conformity in implementations, clarifies the standard itself for implementation, provides a feedback loop to the standards-making bodies for improvements to the standard, and encourages commercial development by providing a baseline for commonality 

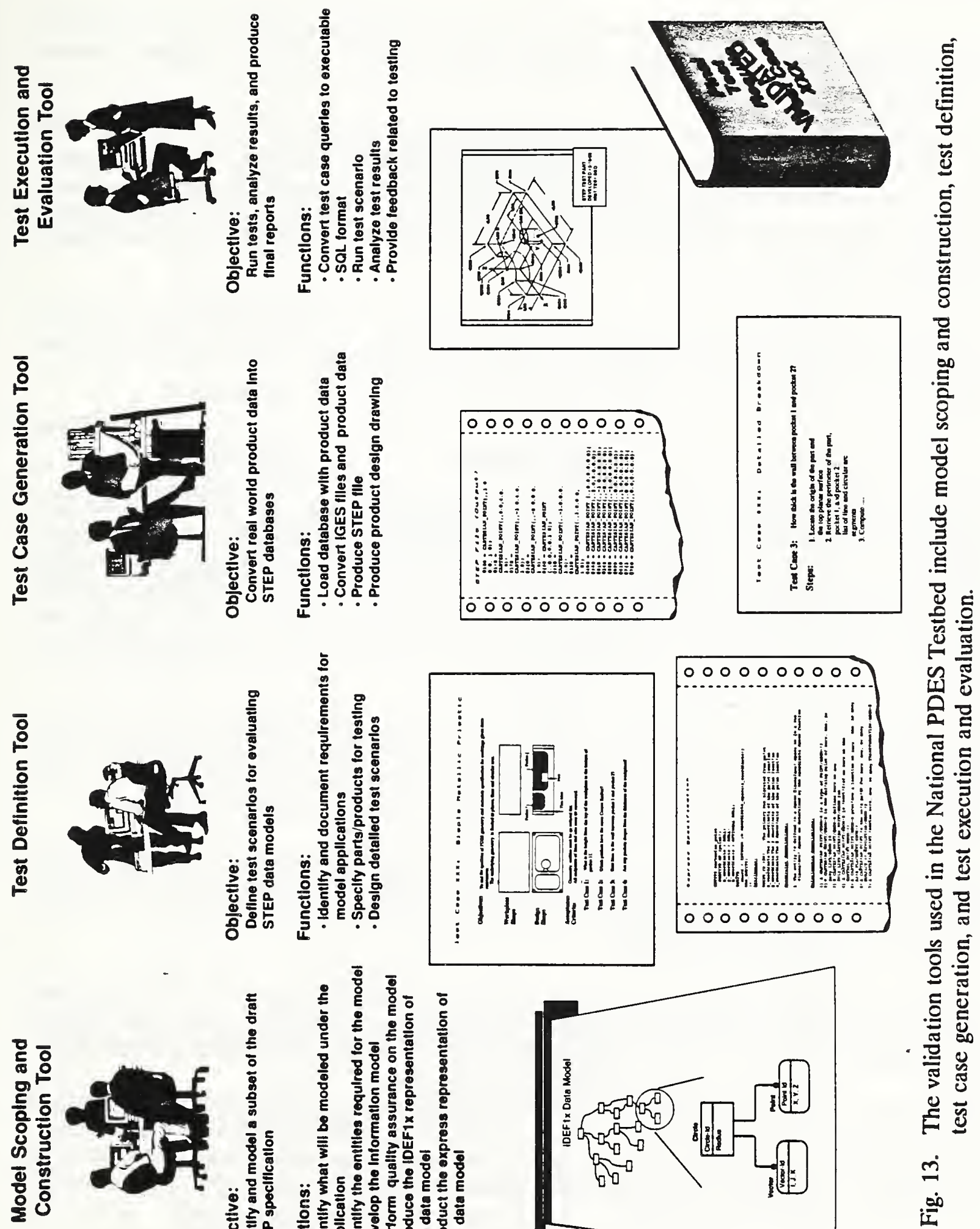
in all products. It does not guarantee that the product conforms to the standard, nor does it assure that the product is of high quality or reliability.

The implementation of a conformance testing system and an independent testing program increases the probability that different STEP implementations will be able to interoperate. Figure 14 shows the conformance testing program model.

The National PDES Testbed will construct a conformance testing system [45]. In cooperation with others, the Testbed plans to develop test procedures and data that adhere to STEP application protocols, specify the process which will be used for certifying compliance with the standard, and define the procedure which will be used to approve and review the operations of testing laboratories. The Testbed intends to help establish a conformance testing program at selected sites on the Product Data Exchange Network.

The standardization and acceptance of a conformance testing methodology, as well as appropriate test methods will allow producers to test their own products through a testing laboratory and will lead to acceptance of test results from different testing laboratories.

\section{Application Prototyping and Interoperability Testing}

For application prototyping and interoperability testing, the Testbed includes a "STEP Production Cell." The STEP Production Cell will demonstrate small batch manufacturing using STEP data [46]. It will be an integrated, automated manufacturing environment within the NIST AMRF whose product specification data representation is based upon validated STEP data models. It will help verify that the STEP standard is workable through production level testing. In cooperation with test sites having similar capabilities, the STEP Production Cell will test and demonstrate how STEP supports production operations occurring at different sites.

The STEP Production Cell will integrate basic STEP software tools, commercial databases, and commercial manufacturing applications into a prototype small-scale manufacturing environment. Within this environment, it will be possible to verify the performance of STEP under real-world conditions and to demonstrate STEP-based manufacturing across different production sites.

The manufacturing data preparation subsystems of the STEP Production Cell are design, process planning, and equipment programming. These subsystems are used to generate the information that is required to control the manufacture and inspection of a part. STEP data is the primary information shared by these subsystems.

Within the cell, the Machining Workstation is a 3-axis vertical milling machine. This computer-driven machine tool can produce simple, prismatic parts. The computer programs that control this machine tool are derived from the STEP data provided by the manufacturing data preparation subsystems.

The inspection workstation, a coordinate measuring machine, provides the facility for determining whether machined parts are produced as specified. Based on measurements from the coordinate measuring machine, analysis software determines whether dimensions of the machined part fall within designed tolerances. As with the milling machine, the computer programs that control the 

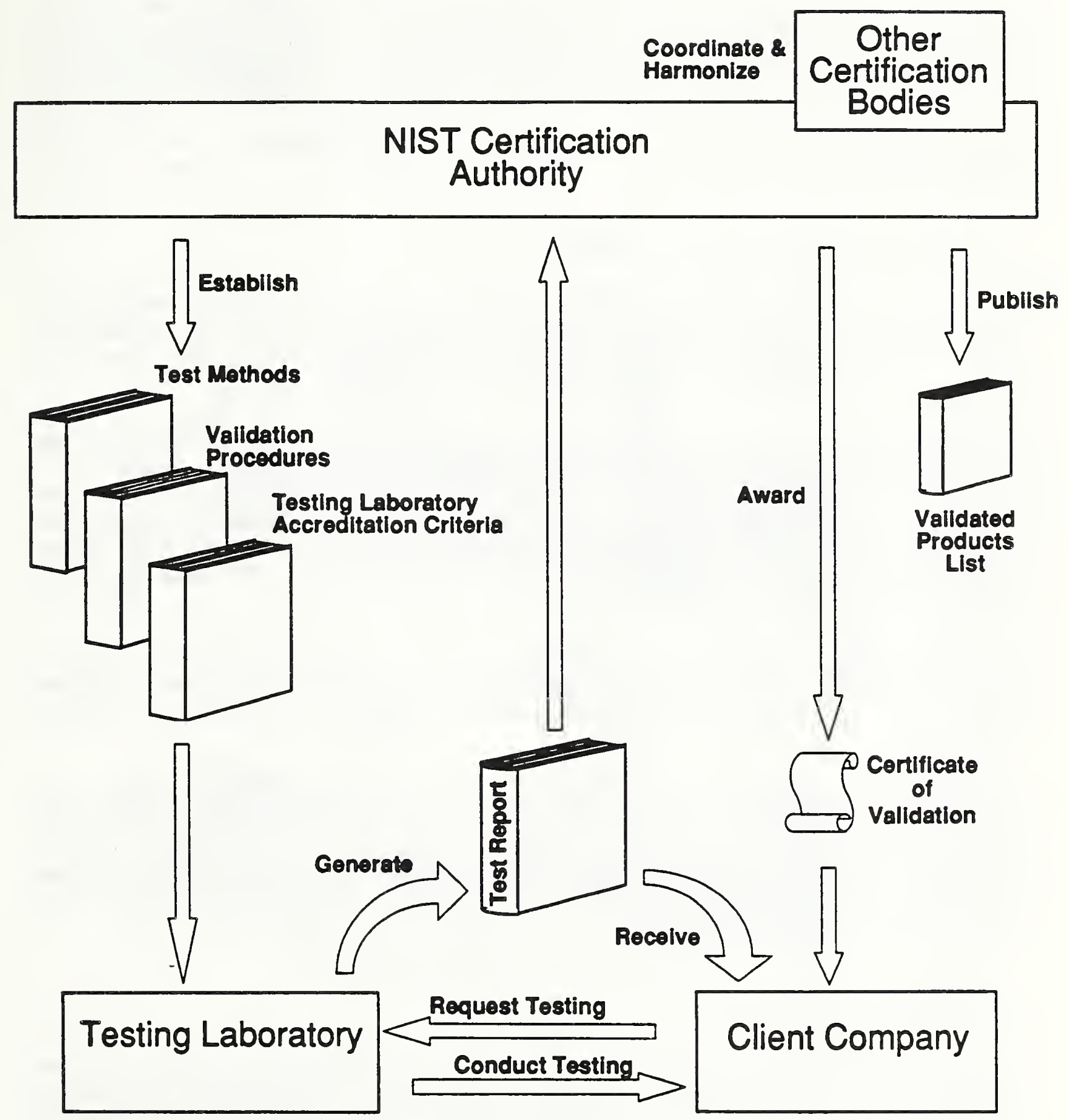

Fig. 14. Conformance testing and certification is a depicted in the conformance testing process model. 
measurement process are derived from the STEP data provided by the manufacturing data preparation subsystems.

The data repository subsystem provides the storage mechanism for STEP data. The repository provides a generic software interface to the data representations. The generic interface allows the application subsystems to store and retrieve the desired STEP data without regard to the details of its representation. The network communications subsystem ties the other six subsystems together.

Figure 15 describes some of the major processes and information contained within the STEP Production Cell.

\section{Product Data Exchange Network Integration}

The Product Data Exchange Network will be a network of organizations and individuals dedicated to support the specification, validation, prototyping, commercial development, and conversion to STEP. The Network will help accelerate the realization of STEP and will help ensure that STEP will function as intended in actual manufacturing environments [47].

The Network will consist of a broad spectrum of manufacturing facilities and research centers from industry, academia, and government linked electronically via computer networks. The plan is to begin with the AMRF-based experience in mechanical parts, then to expand into other areas. Eventually, the Network will include sites in various manufacturing domains, such as aerospace, shipbuilding, apparel, sheet metal products, electrical products, and others. A goal of the Product Data Exchange Network is to accelerate the transition of these facilities to STEP-based information systems.

The National PDES Testbed will serve as headquarters for the Product Data Exchange Network. Because the Network and the CALS Test Network sponsored by the U.S. Department of Defense have similar objectives, the activities and results of these two programs will enhance and complement each other.

Several of the network sites will serve as model facilities for developing STEP-based manufacturing systems. Various Product Data Exchange Network sites will perform STEP validation activities based upon specific capabilities available at that site. These activities may include testing or developing STEP-based software applications, developing transition plans to implement STEP in manufacturing environments, or producing actual products using STEP. Figure 16 depicts some of the activities which may occur at Network sites.

\section{Configuration Management}

The National PDES Testbed provides configuration management systems and services for key organizations participating in major PDES and STEP activities. The Testbed configuration management system can be used to control access and distribution of documents and software. In the future, product models and graphical representations will be included [48]. The functional architecture of the system is shown in Figure 17. 


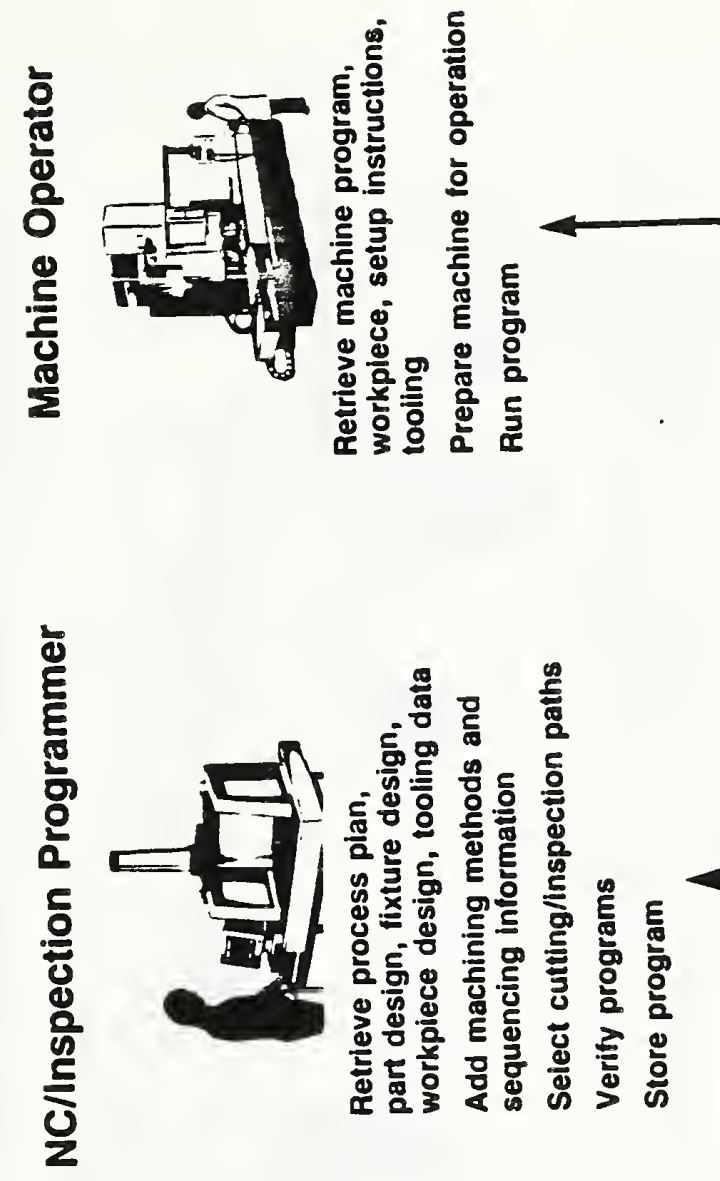

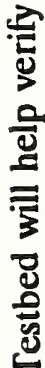

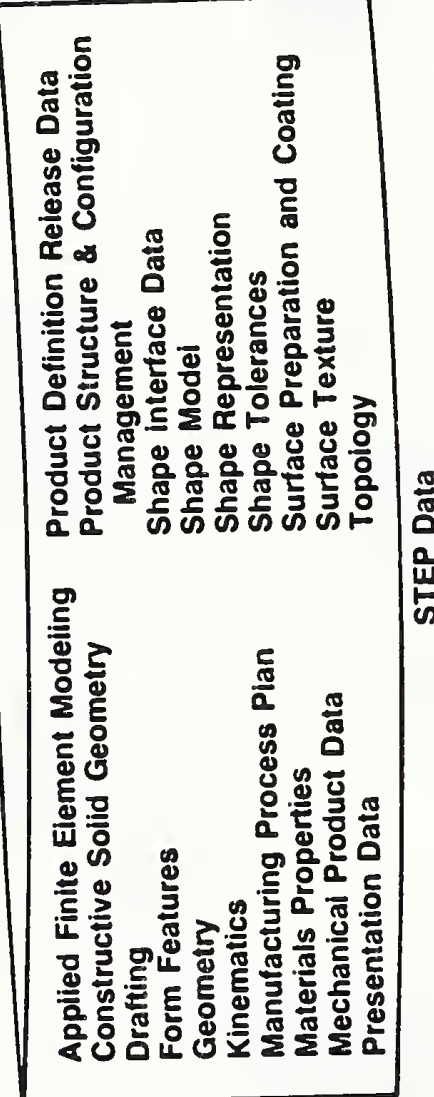

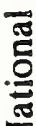

Z

를

능 은

चै

을

될

(2)

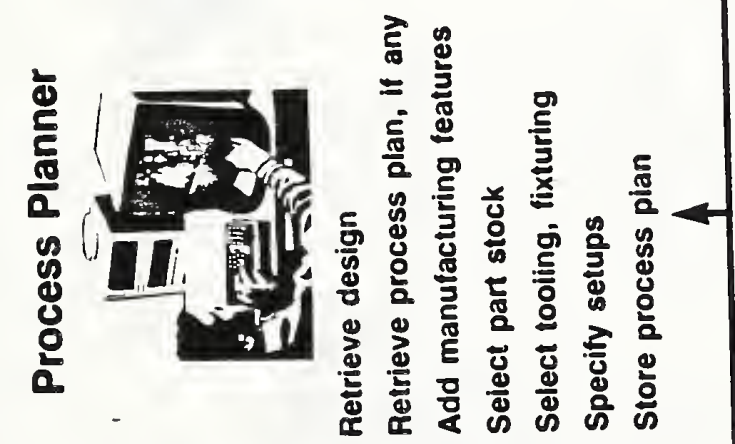

동 은

들 중

芌.

등믄

ฮ ㅀㅗ

额

.

믕

워

ชิ

는

Eِ

ต

舟

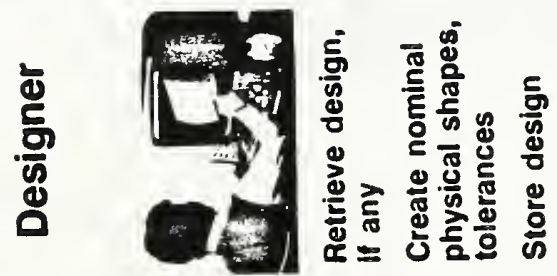



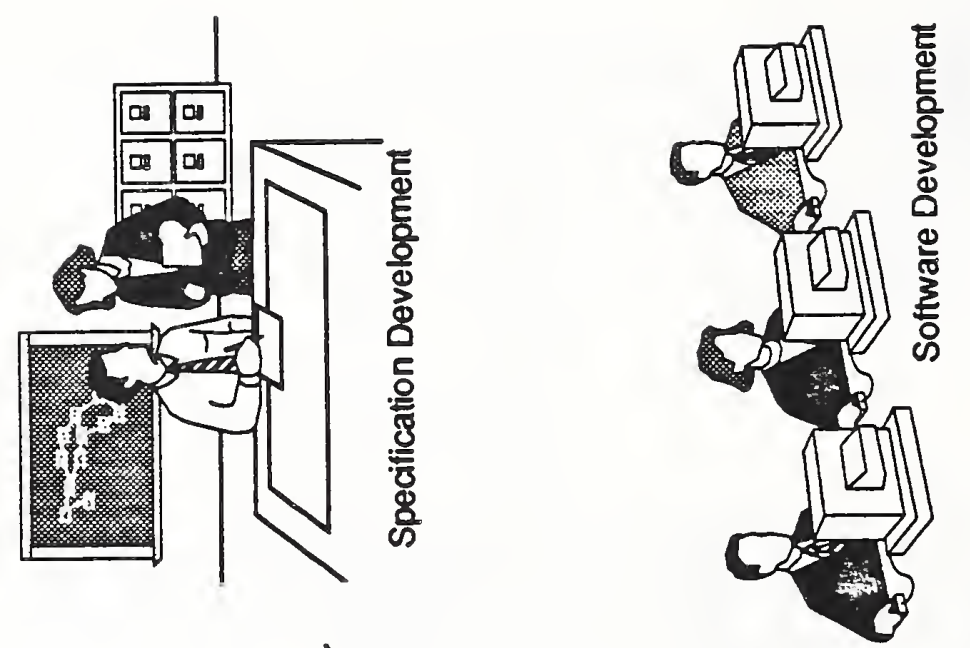

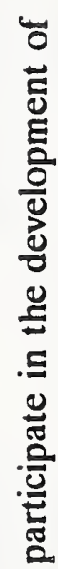
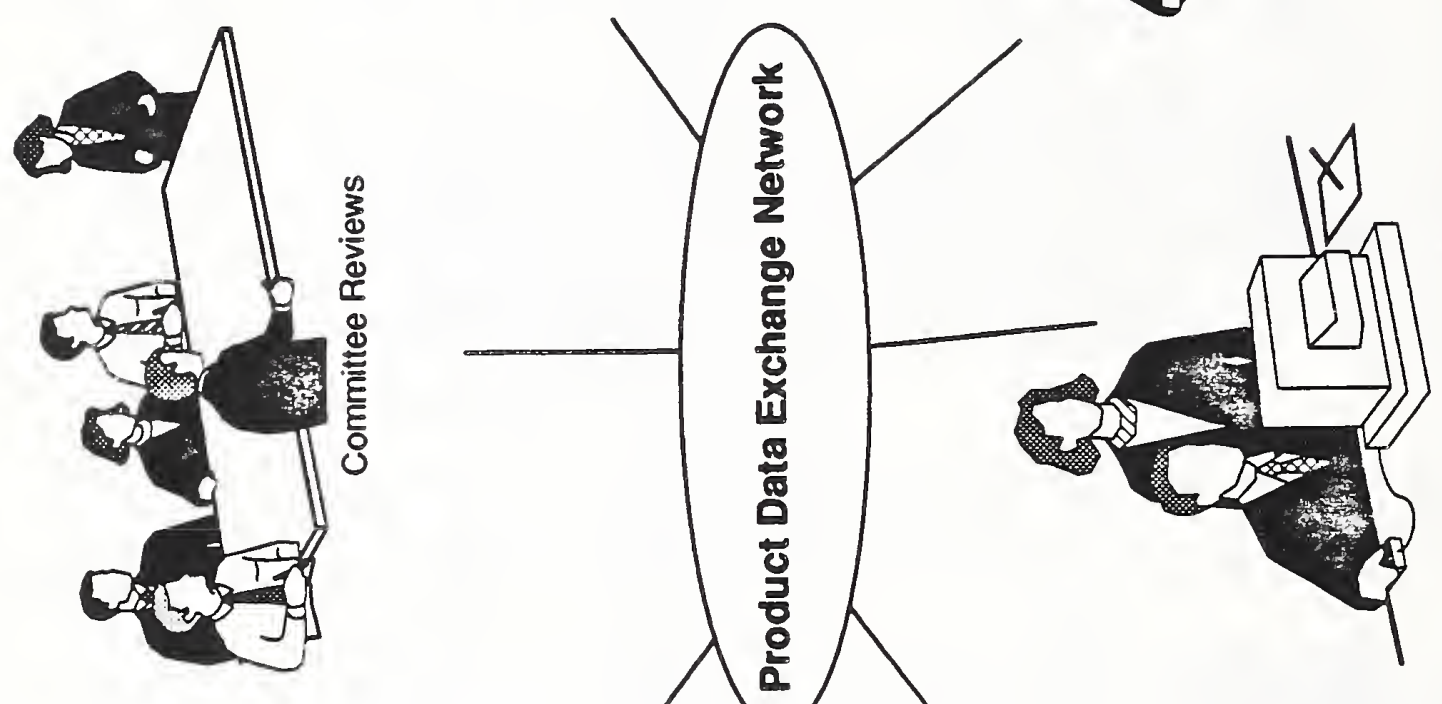

용

号

嗃

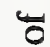

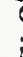

글

ह

产

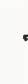

z
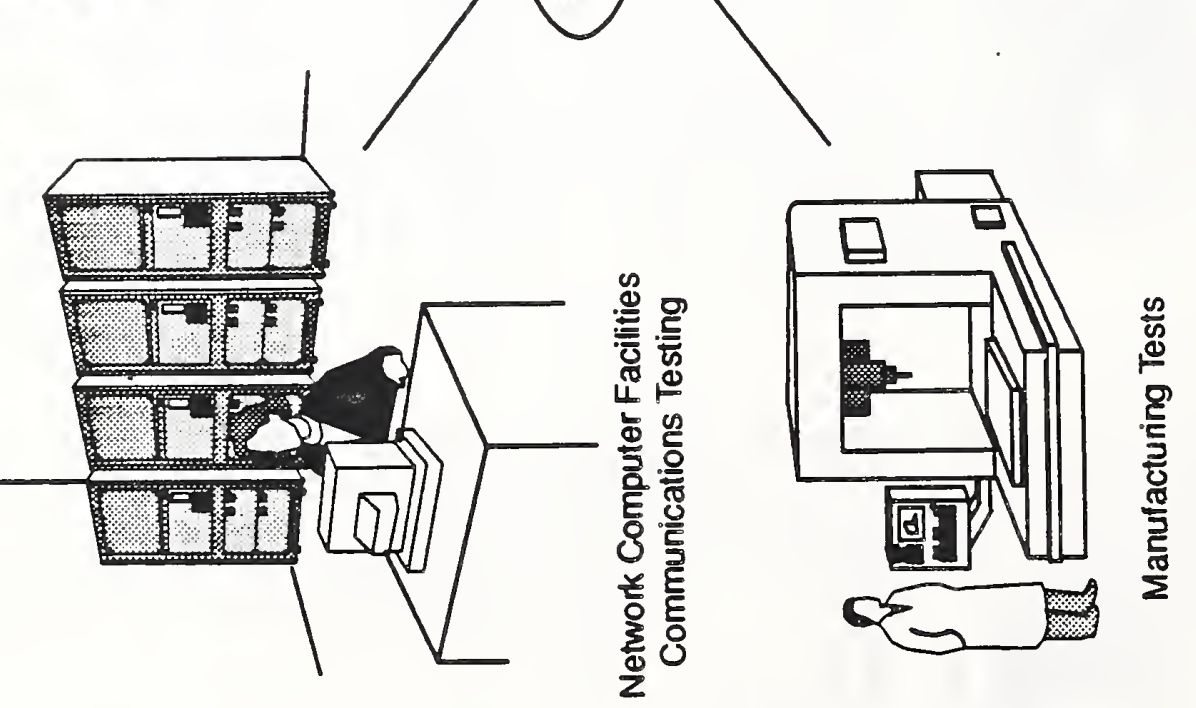

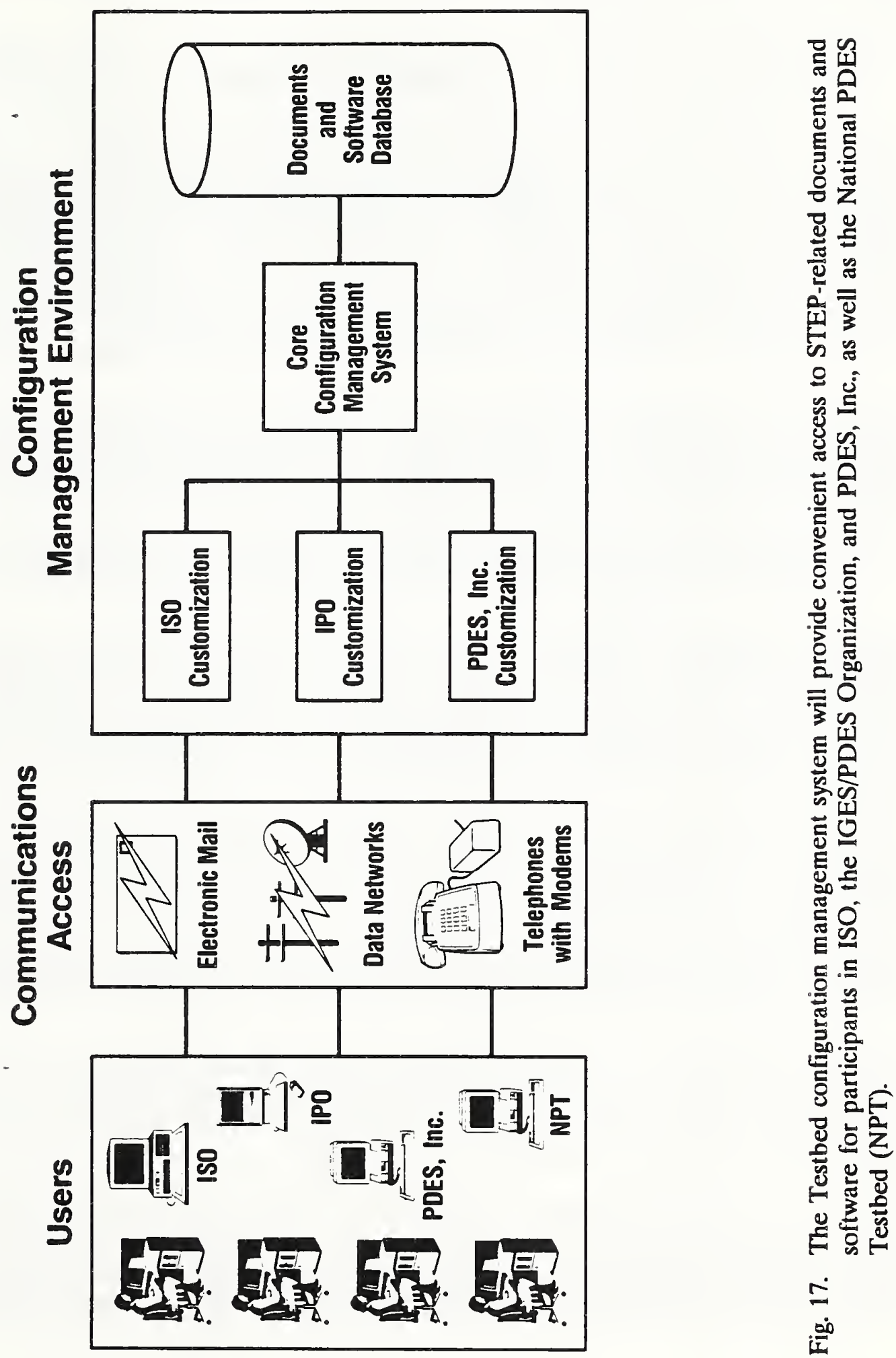
The core of the configuration management system is based upon a general set of common requirements. Customized interfaces will be constructed which account for each organization's internal processes and procedures.

\section{F. The Extension of an Enterprise Integration Framework}

Concurrent engineering is an engineering approach that can help optimize the operations of a manufacturing enterprise. However, the optimization is "localized" to the life cycle--design to production to support--of the enterprise's product. Clearly, concurrent engineering is but one dimension of a bigger idea. That bigger idea is the optimization of all the enterprise's operations, including planning, marketing, and financial operations, as well as its transactions with its suppliers, distributors, and other business partners. "Multi-enterprise concurrent engineering" is the term that connotes the broader optimization. This broader optimization is based upon the integration of all the operations within an enterprise and between an enterprise and its business partners.

The term for the standard architecture that would allow the integration of all activities of manufacturing enterprises is "enterprise integration framework." Just as STEP implies a standard means of representing information about a product as well as the infrastructure necessary to access and contribute to that information in a heterogeneous computer environment,

Enterprise Integration Framework includes the structure, methodologies, and standards to accomplish the integration of all activities of an enterprise.

The key is the sharing of all kinds of information that allows for a concurrent approach not only to engineering, but also to accounting, marketing, management, inventory control, payroll, and other activities that are vital to the functioning of an enterprise. Multi-enterprise concurrent engineering through an enterprise integration framework is an approach that can both guide the integration of an enterprise's activities and provide the standardized organization and arrangement for the integration to occur.

Just as in the implementation of computer integrated manufacturing (CIM) [49], the major technical challenge to an enterprise integrated framework is the design of the integrated system architecture. Beginning with a system architecture, developing the methods to build the models, and then building an integrated framework is the "top down" approach to the integration of all components of an enterprise. A number of architectures have been proposed for CIM [50], but enterprise integration architectures have been studied only recently.

Because every company is unique in the way that it operates and because there are different laws and cultures in different countries that affect how businesses operate, it is essential that an enterprise integration framework be flexible and conceptually broad. This is the realm of enterprise modeling. [51]

Enterprise modeling is the abstract representation, description and definition of the structure, processes, information, and resources of an identifiable business, government activity, or other large entity. The goal of enterprise modeling is to achieve model-driven enterprise integration and operation. Also important are modeling techniques for describing the logistic supply chains in an 
industry, including the business processes that occur among independent but closely cooperating enterprises.

It is also essential that such a large undertaking as enterprise integration framework development be carried out internationally. The consensus development of international standards for integrating enterprises will help assure that the benefits of concurrent engineering approaches, as well as opportunities for global economic competitiveness, are available to all enterprises.

Open Systems Architecture (OSA) is the description of those computing and networking systems that are based on international and de facto public domain standards, rather than the proprietary systems dominating the current business environment. The concept is to be able to create modular information technology components, thus providing for a "plug and play" ability to swap out both hardware and software components among various vendor products. Complex products for OSA require substantial investment and development time. Much of the OSA product planning is precompetitive and linked to standards activities that require coordination.

In the U.S., a number of government agencies are initiating efforts to define and develop an enterprise integration framework. These agencies include the Air Force (through the Wright Research and Development Center's Manufacturing Technology Directorate), the Defense Advanced Research Projects Agency (DARPA), the CALS office under the Office of the Secretary of Defense, and NIST. A major goal is a set of international standards that provide a framework upon which commercial (and government funded) information technology related products could be produced that will support multi-enterprise information systems for industrial applications.

The Air Force Enterprise Integration Framework Program is intended to provide a common reference model for establishing research priorities, harmonizing standards development efforts, and developing a strategy for coordinated investment by government and industry in automated infrastructures. It is anticipated that an international consensus can be built for use of this framework as the model for the development or implementation of international standards and for integrating many types of applications and industries. The program is part of the U.S. effort to cooperate internationally in a coordinated program to define, develop, and validate a conceptual framework for inter- and intra-enterprise integration based on open systems principles and international standards.

Within the European Strategic Program for Research on Information Technology (ESPRIT), a government-industry European CIM Architecture (AMICE) consortium is working to develop a Computer Integrated Manufacturing Open Systems Architecture (CIM OSA) [50], [52].

In a sense, just as multi-enterprise concurrent engineering is the next step in the evolution of manufacturing, the enterprise integration framework is the next step in the evolution of engineering standards. As indicated in Figure 18, engineering education will have to evolve also. Perhaps product data engineering will become as important as the traditional engineering specialties were in the early part of this century.

A vision of the future manufacturing environment is shown in Figure 19. Independent enterprises operating as suppliers, system integrators, merchants and customers are integrated by an information framework into an effective system. Within each of these enterprises, the various product-related 


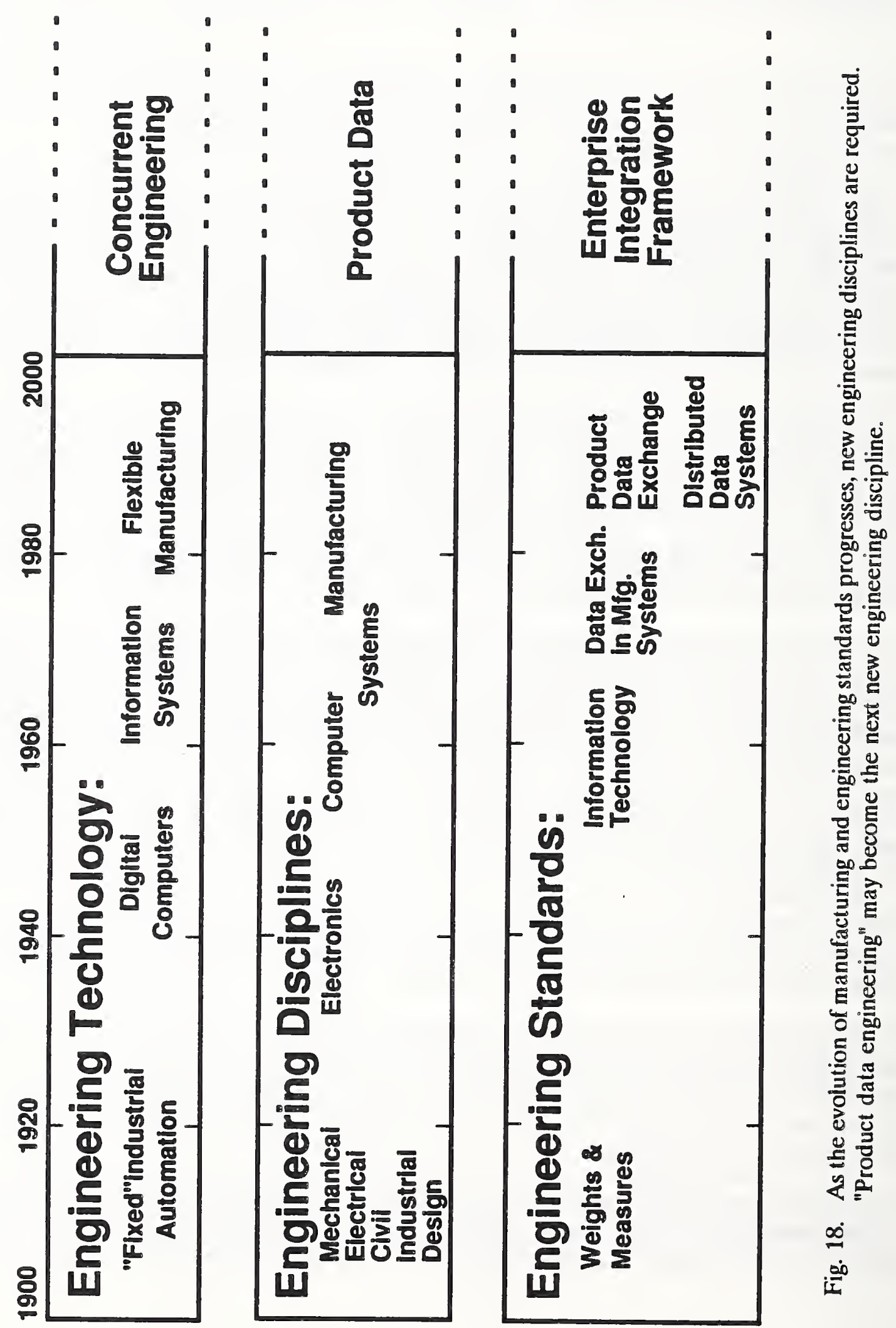




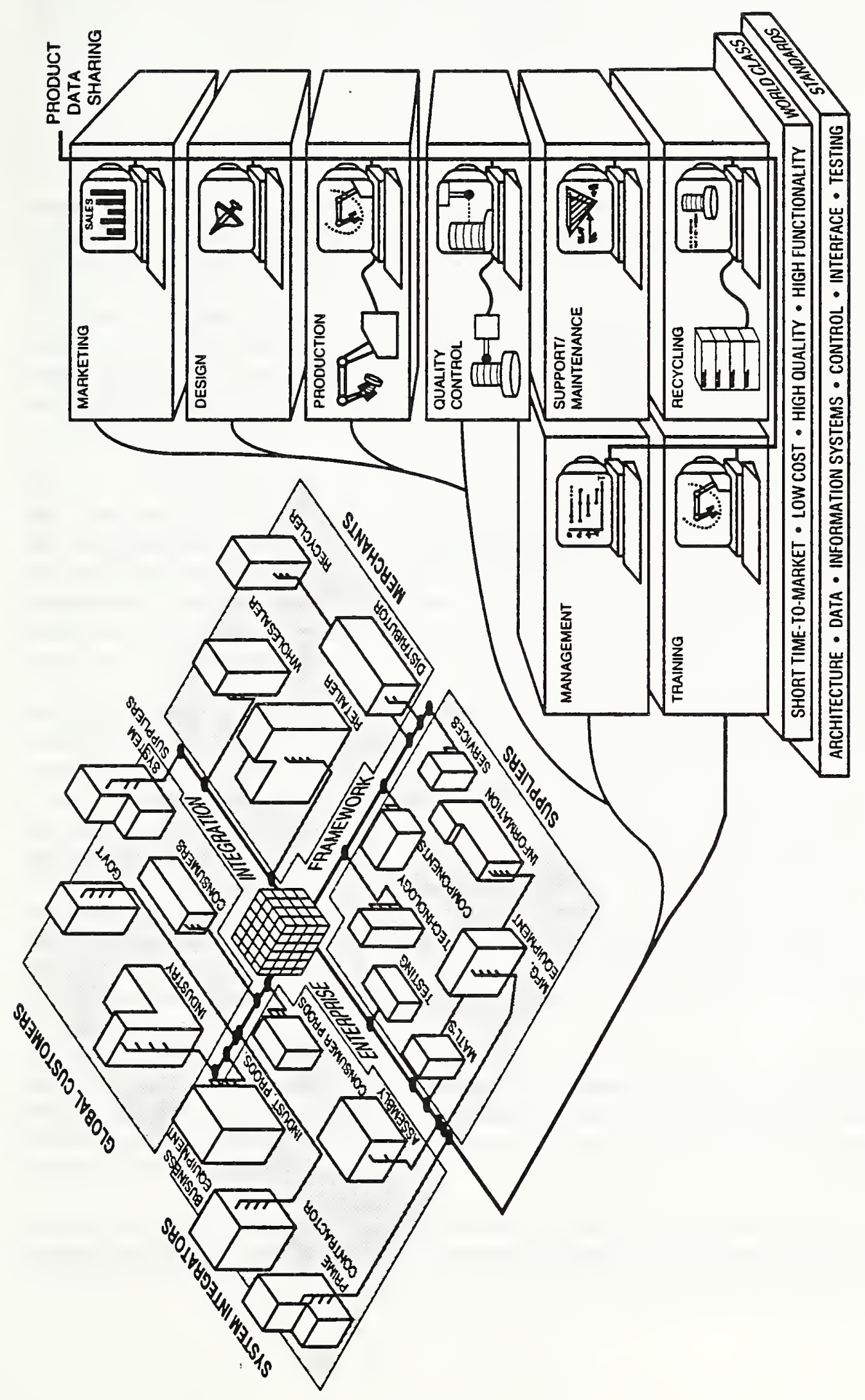

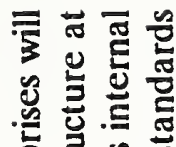

造总站

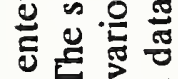

농

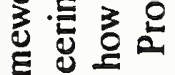

凷高

둬워

可捳业

촌 홍

टु ट्ठ

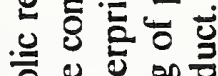

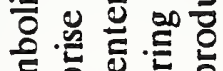

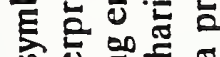

穴尫的䒕

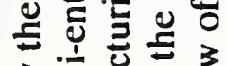

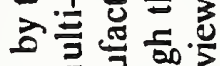

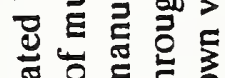

ชुํㅇ

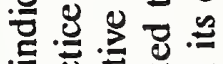

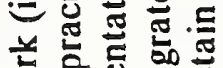

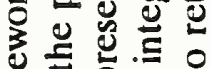

ํํㄴ 은

혼

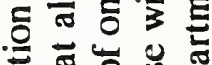

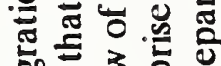

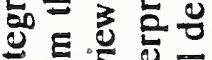

.

. 혼

는 을

츤.

펋 约

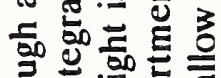

อ.․ㅡ는

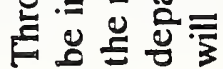

a

里 
functions and product life cycle stages are integrated through the sharing of product data, although each stage maintains its own view of the product. Based upon standards, the inter- and intraenterprise integration enables the practice of multi-enterprise concurrent engineering [53]. It is the practice of multi-enterprise concurrent engineering through which the characteristics of world-class products are achieved. These characteristics are short-time-to-market, low cost, high quality, and high functionality.

\section{CONCLUSIONS}

The primary aim of any manufacturing enterprise is to deliver working products to customers. To this could be added timeliness, cost effectiveness, quality, reliability, and other characteristics that contribute to a competitive product and hence to profits. Nevertheless, the bottom line is simply working products in the hands of satisfied customers. Recently there has been increased recognition that concurrent engineering, engineering design, manufacturing engineering practices, and data exchange and interface standards are critical to international competitiveness [54] [55]. These technologies, based upon information technology in general, are the means for providing to customers high quality and reliable products, as well as the support for those products, in a timely and cost-effective manner.

Information technology will provide an integrated level of automation based upon standards and frameworks. It will create a climate in industry in which enterprises can benefit from cooperation, collaboration and interdependence, without sacrificing their individual independence, initiative, and intellectual property rights. Information technology, by enabling such approaches as concurrent engineering, will stimulate the necessary standardization and provide the economies of scale that would not be otherwise provided without drastic changes in the way businesses in the U.S. operate.

Concurrent engineering, based upon information technology, will initiate a new industrial revolution. Certainly, the bottom line would still be working products in the hands of customers, but future products are much more likely to be of higher quality and more reliable, state-of-the-art products at prices that are much lower than they might have been if concurrent engineering were not used.

It is instructive to reflect on the mechanical drawing and the way it impacted the entire manufacturing process in its era. Prior to the industrial revolution, manufacturing was defined by a physical model of a product to be reproduced. For example, a worker would ensure that the dimensions of the product to be produced corresponded to the model by using calipers to transfer measurements from one to the other. This method reinforced the tradition that workers manufactured complete but specific product types rather than generic components of products.

In 1801, Gaspard Monge wrote "La Geometrie Descriptive." It was the first treatise on modern engineering drawings. It described the concept of projecting dimensioned geometric views of an object onto three perpendicular planes. Since it included size and shape information, the mechanical drawing became an objective standard of performance for workmanship and thus the need for a model was eliminated.

The drawing enabled the practice of designing a product with interchangeable parts. A product could be produced by contractors who could manufacture different components to be assembled. 
This capability led to the fragmentation of the manufacturing process that exists to this day. Moreover, in today's industrial enterprises, the life cycle processes for a product are no longer even performed by the same group of people. In fact, the processes are distributed through a network of factories.

The mechanical drawing concept has lasted for almost 200 years. Although it is a method for describing products, just as the physical model had been, the mechanical drawing revolutionized the manufacturing process itself. The drawing became the output of the design phase of the process and the input into the production phase. Drawings were converted into production process plans which were converted into programs or procedures for all the manufacturing operations. Every step of the manufacturing process has its own view of the product data. These dissimilar views make it difficult to return to the designer evaluative or corrective knowledge about the different processes.

As we move into the twenty-first century, new manufacturing technologies are needed to improve productivity and competitiveness. In our information and computer age, companies exchange and share information across the country. This capability is needed for manufacturing today's complex products such as automobiles, airplanes, ships, and buildings.

Multi-enterprise concurrent engineering will require the ability to store and retrieve product data far beyond the capability of the mechanical drawing. The replacement for the mechanical drawing that will allow revolutionary new engineering technologies is product data sharing. This new capability will make available to the designer knowledge about all other processes. It will process product data through automated computer-based techniques that allow for shared access among the life cycle processes in support of concurrent engineering. It will make available an integrated product data model that allows access to multiple views of the product.

STEP, as well as other new product data, data exchange and interface standards and their supporting technologies, must be implemented for this new product data sharing capability to be successful. That is why concurrent engineering is impossible without standards.

The critical concept is this: standards for product data and data exchange are important because they enable and facilitate an automated form of concurrent engineering that can be implemented in a computerized environment. This automated, or computer-aided, concurrent engineering provides a mechanism for multi-enterprise integration. As a result, the automated practice of concurrent engineering among manufacturing enterprises, their customers and their suppliers, including suppliers of technology as well as materials and components, would create a new kind of multi-enterprise concurrent engineering. This kind of multi-enterprise concurrent engineering could be achieved without the surrendering of historical forms of personal interactions currently practiced by workers and managers.

Accordingly, an automated approach to multi-enterprise concurrent engineering could be especially valuable in the commercial environment of the U.S. It could merge the many dynamic and innovative, mostly small, entrepreneurial companies along with larger manufacturing enterprises into an integrated and cooperative group

Yet although they would be integrated in the way they contribute their talents to the life cycles of products, participants in such diverse groups could remain individualistic and independent in the way they operate and manage their businesses. Although participating companies would work in an 
integrated fashion, and enjoy the benefits of concurrent engineering, their ability to retain their individual freedoms would preserve for them the benefits associated with the traditional strengths of U.S. commercial and individual diversity.

In these ways, multi-enterprise concurrent engineering could match the historically successful style of entrepreneurial innovation in the U.S. with the competitive and economic demands of today's global economy. The result could be the reemergence of U.S. manufacturing in world markets.

\section{ACKNOWLEDGEMENTS}

A substantial number of the concepts and results discussed in this chapter represent the accomplishments of staff members of the Factory Automation Systems Division in the Manufacturing Engineering Laboratory, and of other divisions at NIST. We are grateful for their technical contributions, as well as their leadership efforts in ensuring that manufacturing data interface standards will indeed become a reality. Many members of our Division provided us with information about their work for this chapter. We are grateful for their assistance. We especially thank, for their more direct contributions to our manuscript, Charles R. McLean, Manager of the CALS/PDES Project, which includes the NIST National PDES Testbed; Peter Brown, Manager of the Engineering Design Laboratory; and Cita Furlani, Leader of the Product Data Engineering Group, all in our Division. Also, we thank Sharon J. Kemmerer, who is in the Information Systems Engineering Division of the NIST Computer Systems Laboratory, for her many valuable suggestions that improved our manuscript.

\section{REFERENCES}

1. R. I. Winner, J. P. Pennell, H. E. Bertrand, and M. M. G. Slusarczuk, "The Role of Concurrent Engineering in Weapons System Acquisition," Institute for Defense Analyses, Report R-338, 175 pages, (December 1988).

2. "America's Choice: High Skills or Low Wages," Commission on Skills of the American Workforce, I. C. Magaziner, Chair (1990). (Available from National Center on Education and the Economy, 39 State Street, Suite 500, Rochester, NY 14614.)

3. A. Lowenstein and S. Schlosser, "DOD/Industry Guide to Automation of a Concurrent Engineering Process," Prospective Computer Analysts, Inc. Report CE-Guide, 114 pages (December 1988). (Available from Prospective Computer Analysts, Inc., 1800 Northern Blvd., Roslyn, NY 11576.)

4. J. L Nevins and D. E. Whitney, eds., "Concurrent Design of Products and Processes: A Strategy for the Next Generation In Manufacturing," McGraw-Hill, New York, 1989.

5. "Application of Concurrent Engineering to Mechanical Systems Design," CALS Industry Steering Group, Technical Report 002, 97 pages (June 16, 1989). (Available from the National Security Industrial Association, Attn: CALS Industry Steering Group, Suite 300, 1025 Connecticut Ave. NW, Washington, DC 20036.) 
6. C. W. Kelly, and J. L. Nevins, "Findings of the U.S. Department of Defense Technology Assessment Team on Japanese Manufacturing Technology," The Charles Stark Draper Laboratory, Report R-2161, for the Defense Advanced Research Agency/Information Science and Technology Office, Procurement Number MDA972-C-0027, 209 pages (June 1989).

7. H. M. Bloom, "The Role of the National Institute of Standards and Technology As It Relates To Product Data Driven Engineering," National Institute of Standards and Technology, Interagency Report 89-4078, 35 pages (April 1989). (Available from the National Technical Information Service (NTIS), Springfield, VA 22161.)

8. "A Framework for Concurrent Engineering," CALS Industry Steering Group (to be published). (Available from the National Security Industrial Association, Attn: CALS Industry Steering Group, Suite 300, 1025 Connecticut Ave. NW, Washington, DC 20036.)

9. J. Naisbitt, "Megatrends: Ten New Directions Transforming Our Lives," Warner Books, New York (1982).

10. "Emerging Technologies: A survey of Technical And Economic Opportunities," Technology Administration, U.S. Department of Commerce, 55 pages (Spring 1990).

11. "The Competitive Status of the U.S. Electronics Sector from Materials to Systems," International Trade Administration, U.S. Department of Commerce, 221 pages (April 1990). (Available from the Superintendent of Documents, U.S. Government Printing Office, Washington, DC 20402-9325.)

12. "Making Things Better: Competing in Manufacturing," Office of Technology Assessment, Congress of the United States, OTA-ITE-443, 241 pages (February 1990). (Available from the Superintendent of Documents, U.S. Government Printing Office, Washington, DC 204029325.

13. K. B. Clark, W. B. Chew, and T. Fujimoto, "Product Development in the World Auto Industry: Strategy, Organization, Performance," Presentation to the Brookings Institution Microeconomics Conference, December 3, 1987. (Available from the Graduate School of Business Administration, Harvard University.)

14. C. H. Ferguson, "Computers and the Coming of the U.S. Keiretsu," Harvard Business Review 68, No. 4, 55-70, (July-August 1990).

15. D. L. Wince-Smith, in "Debate: Can a Keiretsu Work in America?," Harvard Business Review 68, No. 5, 197 (September-October 1990).

16. W. M. Henghold, G. C. Shumaker, and L. Baker, Jr., "Considerations for the Development and Implementation of PDES Within a Government Environment," Manufacturing Technology Directorate, Air Force Wright Aeronautical Laboratories, Report AFWAL-TR89-8009, 145 pages (February 1989).

17. "Computer-aided Acquisition and Logistic Support (CALS) Program Implementation Guide," Department of Defense, Military Handbook MIL-HDBK-59A, September 1990. 
18. K A. Reed, D. Harrod, and W. Conroy, "The Initial Graphics Exchange Specification, Version 5.0," National Institute of Standards and Technology, Interagency Report 4412 (September 1990). (Available from the National Technical Information Service (NTIS), Springfield, VA 22161.)

19. "IGES/PDES Organization: Reference Manual," National Computer Graphics Association, Fairfax, VA, July 1990.

20. "STEP Part 1: Overview and Fundamental Principles," ISO TC184/SC4/WG1, Document N494 Version 3 (May 1990).

21. C. Furlani, J. Wellington, and S. Kemmerer, "Status of PDES-Related Activities (Standards and Testing)," National Institute of Standards and Technology, Interagency Report 4432, 18 pages (October 1990). (Available from the National Technical Information Service (NTIS), Springfield, VA 22161.)

22. "A Strategy for Implementing a PDES/STEP Data Sharing Environment," PDES, Inc. (to be published). (Available from the South Carolina Research Authority, Trident Research Center, 5300 International Blvd., N. Charleston, SC 29418.)

23. "EXPRESS Language Reference Manual," ISO TC184/SC4/WG1, Document N496 (May 1990).

24. "The STEP File Structure," ISO TC184/SC4/WG1, Document N279 (September, 1988).

25. "Common Data Model Subsystem, Data Model Subsystem, Vol. 5, Part 4, Information Modeling Manual IDEF1-Extended (IDEF1X)," Manufacturing Technology Directorate, Air Force Wright Aeronautical Laboratories, Report AFWAL-TR-86-4006 (1986).

26. S. N. Clark, "An Introduction to the NIST PDES Toolkit," National Institute of Standards and Technology, Interagency Report 4336, 8 pages (May 1990). (Available from the National Technical Information Service (NTIS), Springfield, VA 22161.)

27. M. Palmer, and M. Gilbert, "Guidelines for the Development and Approval of Application Protocols," Working Draft, Version 0.7 ISO TC184/SC4/WG4, Document N1 (February 1991).

28. M. Mitchell, "Testbed Development Plan: Validation and Testing System Development," National Institute of Standards and Technology, Interagency Report 4417, 38 pages (September 1990). (Available from the National Technical Information Service (NTIS), Springfield, VA 22161.)

29. "Bylaws of the Digital Representation of Product Data Standards Harmonization Organization," ANSI (to be published). (Available from the American National Standards Institute, 1430 Broadway, New York, NY 10018.)

30. M. Mitchell, Y. Yang, S. Ryan, and B. Martin, "Data Model Development and Validation for Product Data Exchange," National Institute of Standards and Technology, Interagency Report 
90-4241, 13 pages (January 1990). (Available from the National Technical Information Service (NTIS), Springfield, VA 22161.)

31. M. J. McLay and K. C. Morris, "The NIST STEP Class Library (STEP into the Future)," National Institute of Standards and Technology, Interagency Report 4411, 20 pages (August 1990). (Available from the National Technical Information Service (NTIS), Springfield, VA 22161.)

32. C. Stark, and M. Mitchell, "Development Plan: Application Protocols for Mechanical Parts Production," National Institute of Standards and Technology, Interagency Report (to be published). (Available from the National Technical Information Service (NTIS), Springfield, VA 22161.)

33. W. F. Danner, "A Proposed Integration Framework for STEP (Standard for the Exchange of Product Model Data)," National Institute of Standards and Technology, Interagency Report 90-4295 (April 1990). (Available from the National Technical Information Service (NTIS), Springfield, VA 22161.)

34. W. F. Danner, and Y. Yang, "Generic Product Data Model (GPDM)," National Institute of Standards and Technology, Interagency Report, (to be published); also, "Generic Enterprise Data Model (GEDM)," National Institute of Standards and Technology, Interagency Report, (to be published). (Available from the National Technical Information Service (NTIS), Springfield, VA 22161.)

35. C. R. McLean, "Interface Concepts for Plug-Compatible Production Management Systems," Proceedings of the IFIP W.G. 5.7: Information Flow in Automated Manufacturing Systems, Gaithersburg, MD, August 1987; reprinted in Computers in Industry 9, 307-318 (1987).

36. H. M. Bloom, C. Furlani, M. Mitchell, J. Tyler, and D. Jefferson, "Information Resource Dictionary System: An Integration Mechanism for Product Data Exchange Specification," National Institute of Standards and Technology, Interagency Report 88-3862, 15 pages (October 1988). (Available from the National Technical Information Service (NTIS), Springfield, VA 22161.)

37. T. R. Kramer, "Enhancements to the VWS2 Data Preparation Software," National Institute of Standards and Technology, Interagency Report 89-4201, 58 pages (November 1989). (Available from the National Technical Information Service (NTIS), Springfield, VA 22161.)

38. H. T. Moncarz, "Architecture and Principles of the Inspection Workstation," National Institute of Standards and Technology, Interagency Report 88-3802, 46 pages (September 1990). (Available from the National Technical Information Service (NTIS), Springfield, VA 22161.)

39. J. S. Tu and T. H. Hopp, "Part Geometry Data in the AMRF," National Institute of Standards and Technology, Interagency Report 87-3551, 16 pages (April 1987). (Available from the National Technical Information Service (NTIS), Springfield, VA 22161.) 
40. D. Libes and E. J. Barkmeyer, "The Integrated Manufacturing Data Administration System (IMDAS)--An Overview," International Journal of Computer Integrated Manufacturing 1, 44-49 (1988).

41. S. Rybczynski, E. J. Barkmeyer, E. K. Wallace, M. L. Strawbridge, D. E. Libes, and C. V. Young, "AMRF Network Communications," National Institute of Standards and Technology, Interagency Report 88-3816, 206 pages (June 1988). (Available from the National Technical Information Service (NTIS), Springfield, VA 22161.)

42. A. B. Feeney, "Engineering Design Laboratory Guide," National Institute of Standards and Technology, Interagency Report 4519, 13 pages (February 1991). (Available from the National Technical Information Service (NTIS), Springfield, VA 22161.)

43. Y. T. Lee, "On Extending the Standard for the Exchange of Product Data to Represent TwoDimensional Apparel Pattern Pieces," National Institute of Standards and Technology, Interagency Report 4358, 27 pages (June 1990). (Available from the National Technical Information Service (NTIS), Springfield, VA 22161.)

44. C. R. McLean, "National PDES Testbed Strategic Plan 1990," National Institute of Standards and Technology, Interagency Report 4438, 73 pages (October 1990). (Available from the National Technical Information Service (NTIS), Springfield, VA 22161.)

45. S. J. Kemmerer, "Development Plan: Conformance Testing System," National Institute of Standards and Technology, Interagency Report (to be published). (Available from the National Technical Information Service (NTIS), Springfield, VA 22161.)

46. J. E. Fowler, "STEP Production Cell Technical Development Plan," National Institute of Standards and Technology, Interagency Report 4421, 29 pages (September 1990). (Available from the National Technical Information Service (NTIS), Springfield, VA 22161.)

47. S. P. Frechette and K. Jurrens, "Development Plan: Product Data Exchange Network," National Institute of Standards and Technology, Interagency Report 4431, 27 pages (September 1990). (Available from the National Technical Information Service (NTIS), Springfield, VA 22161.)

48. S. Ressler and S. Katz, "Development Plan: Configuration Management Sysiems and Services," National Institute of Standards and Technology, Interagency Report 4413, 28 pages (September 1990). (Available from the National Technical Information Service (NTIS), Springfield, VA 22161.)

49. A. Jones, E. Barkmeyer, and W. Davis, "Issues in the Design and Implementation of a System Architecture for Computer Integrated Manufacturing," Int. J. Computer Integrated Manufacturing 2, No. 2., 65-76 (1989).

50. A. Jones, ed., "Proceedings of CIMCON '90," National Institute of Standards and Technology, Special Publication 785, 528 pages (May 1990). (Available from the National Technical Information Service (NTIS), Springfield, VA 22161.) 
51. A.-W. Scheer, "Enterprise-Wide Data Modelling," Springer-Verlag, Berlin 1989.

52. "Open System Architecture for CIM," Springer-Verlag, Berlin 1989. (Available from ESPRIT Consortium AMICE, 489 Avenue Louise, Bte. 14, B-1050 Brussels, Belgium.)

53. "US TAG to ISO TC 184: Industrial Automation Systems," US TAG to ISO TC 184, Document N 244 (August, 1989).

54. "Improving Engineering Design: Designing for Competitive Advantage," Committee on Engineering Design Theory and Methodology, Manufacturing Studies Board, Commission on Engineering and Technical Systems, National Research Council (1991). (Available from National Academy Press, 2101 Constitution Ave., Washington, DC 20418.)

55. "Report of the National Critical Technologies Panel," National Critical Technologies Panel, W. D. Phillips, Office of Science and Technology Policy, Chair (1991). (Available from The National Critical Technologies Panel, 1101 Wilson Boulevard, Suite 1500, Arlington, VA 22209.) 



\begin{tabular}{|c|c|c|}
\hline \multirow{4}{*}{$\begin{array}{l}\text { NIST-114A } \\
\text { (REV. 3-90) }\end{array}$} & \multirow{4}{*}{$\begin{array}{l}\text { U.S. DEPARTMENT OF COMMERCE } \\
\text { NATIONAL INSTITUTE OF STANDARDS AND TECHNOLOGY }\end{array}$} & $\begin{array}{l}\text { 1. PUBLCATION OR AEPORT NUMBEA } \\
\text { NISTIR } 4573\end{array}$ \\
\hline & & 2. PEAFORMING ORGANIZATION REPORT NUMBER \\
\hline & & 3. PUBLCATION DATE \\
\hline & & May 1991 \\
\hline
\end{tabular}

4. TITLE AND SUBTITLE

Concurrent Engineering Through Product Data Standards

5. AUTHOR(S)

Gary P. Carver, Howard M. Bloom

6. PERFORMINQ ORCANIZATION (IF JOINT OR OTHER THAN NIST, SEE INSTAUCTIONS)

U.S. DEPARTMENT OF COMMERCE

MATIONAL INSTITUTE OF STANDAROS ANO TECHMOLOGY

GATTHEASBURG, MD 20990

7. CONTAACT/GAANT NUMBEA

8. TYPE OF REPORT AND PERIOD COVERED

9. SPONSORING ORGANIZATION MAME AND COMPLETE ADDRESS (STAEET, CITY, STATE, ZIP)

10. SUPPLEMENTAAY NOTES

11. ABSTRACT (A 200-WOAD OR LESS FACTUAL SUMMAAY OF MOST SIONIFICANT INFOAMATION. IF DOCUMENT INCLUDES A SIGNIFICANT BIBUOGAAPHY OR UTERATURE SUAVEY, MENTION IT HERE)

Concurrent engineering involves the integration of people, systems and information into a responsive, efficient system. Integration of computerized systems allows additional benefits: automatic knowledge capture during development and lifetime management of a product, and automatic exchange of that knowledge among different computer systems. Critical enablers are product data standards and enterprise integration frameworks. A pioneering assault on complex technical challenges is associated with the emerging international Standard for The Exchange of Product Model Data (STEP). Surpassing in scope previous standards efforts, the goal is complete, unambiguous, computer-readable definition of physical and functional characteristics of a product throughout its life cycle. U.S. government, industrial, and standards organizations are cooperating in a program, Product Data Exchange using STEP (PDES), to develop and implement STEP in a shared-database environment. PDES will lead to higher, integrated levels of automation based upon information standards and frameworks. U.S. manufacturers will benefit from concurrent engineering without sacrificing strengths and traditions of individuality, initiative, and intellectual property rights. Concurrent engineering, through information technology and standards, represents the power of a new industrial revolution. The role of the NIST National PDES Testbed, technical leadership and testing-based foundation for the development of STEP, is described.

12. KEYWORDS (6 TO 12 ENTRIES; ALPHABETICAL ORDER; CAPITAUZE ONLY PROPEA MAMES; AND SEPARATE KEY WORDS 8Y SEMICOLONS) automated manufacturing; concurrent design; concurrent engineering; information standards; information standards; manufacturing standards; PDES; product data engineering; product data sharing; product data standards; STEP

13.

13. AVALLABILTY

UNEMTTED

FOR OFFICIAL DISTRIBUTION. DO MOT PELEASE TO NATIONAL TECHMICAL INFORMATION SERVICE (NTIS).

ORDER FROM SUPERINTENDENT OF DOCUMENTS, U.S. GOVERNMENT PRINTING OFFICE, WASHINOTON, DC 20402

ORDER FROM NATIONAL TECHMICAL INFOAMATION SEAVCE (NTIS), SPRIMGFIELD, VA 22161.

14. NUMBER OF PAINTED PAGES

76

15. PAICE

A05 


